\title{
Spectroelectrochemistry and Nature of Charge Carriers in Self-Doped Conducting Polymer
}

Anton Volkov, Sandeep Kumar Singh, Eleni Stavrinidou, Roger Gabrielsson, Felipe Franco Gonzalez, Alex Cruce, Weimin Chen, Daniel Simon, Magnus Berggren and Igor Zozoulenko

The self-archived version of this journal article is available at Linköping University Electronic Press:

http:// urn.kb.se/ resolve?urn=urn:nbn:se:liu:diva-140056

N.B.: When citing this work, cite the original publication.

Volkov, A., Kumar Singh, S., Stavrinidou, E., Gabrielsson, R., Franoo Gonzalez, F., Cruce, A., Chen, W., Simon, D., Berggren, M., Zozoulenko, I., (2017), Spectroelectrochemistry and Nature of Charge Carriers in Self-Doped Conducting Polymer, ADVANCED ELECTRONIC MATERIALS, 3(8),. https:// doi.org/ 10.1002/ aelm.201700096

Original publication available at:

https:// doi.org/ 10.1002/ aelm.201700096

Copyright: Wiley: 12 months

http://eu.wiley.com/WileyCDA/ 
DOI: $10.1002 /(($ please add manuscript number $))$

\section{Article type: Full Paper}

\section{Spectroelectrochemistry and nature of charge carriers in self-doped conducting polymer}

Anton V. Volkov, Sandeep Kumar Singh, Eleni Stavrinidou, Roger Gabrielsson, Juan F.

Franco-Gonzalez, Alex Cruce, Weimin M. Chen, Daniel T. Simon, Magnus Berggren and Igor V. Zozoulenko*

Anton V. Volkov, Dr. Sandeep Kumar Singh, Dr. Eleni Stavrinidou, Dr. Roger Gabrielsson, Dr. Juan F. Franco-Gonzalez, Assoc. Prof. Daniel T. Simon, Prof. Magnus Berggren, Prof. Igor V. Zozoulenko

E-mail: igor.zozoulenko@liu.se

Laboratory of Organic Electronics, Department of Science and Technology

Linköping University, 60174 Norrköping

Sweden

Dr. Alex Cruce, Prof. Weimin M. Chen,

Department of Physics, Chemistry and Biology

Linköping University, 58183 Linköping

Sweden

Keywords: self-doped conducting polymers; sodium salt of bis[3,4-

ethylenedioxythiophene]3 thiophene butyric acid (ETE-S); spectroelectrochemistry; polarons, bipolarons; density-functional theory.

\section{Abstract:}

A recently developed water soluble self-doped sodium salt of bis[3,4ethylenedioxythiophene]3thiophene butyric acid (ETE-S) has been electropolymerized and characterized by means of spectroelectrochemistry, electron paramagnetic resonance spectroscopy, and cyclic voltammetry, combined with the density functional theory (DFT) and time dependent DFT calculations. The focus of the studies was to underline the nature of the charge carriers when the electrochemically polymerized ETE-S films undergo a reversible transition from reduced to electrically conductive oxidized states. Spectroelectrochemistry shows clear distinctions between absorption features from reduced and charged species. In the reduced state the absorption spectrum of ETE-S electropolymerized film shows a peak that is attributed to HOMO $\rightarrow$ LUMO transition. As the oxidation level increases, this peak 


\section{WILEY-VCH}

diminishes and the absorption of the film is dominated by spinless bipolaronic states with some admixture of polaronic states possessing a magnetic momentum. For fully oxidized samples the bipolaronic states fully dominate, and the features in the absorption spectra are related to the drastic changes of the band structure exhibiting a strong decrease of the band gap when a polymeric film undergoes oxidation.

\section{Introduction}

In recent years, conducting polymers have become the materials of choice for many bioelectronics applications. ${ }^{[1]}$ Recently, the concept of electronic plants (e-Plants) has emerged as an organic bioelectronics technology capable of forming analogue and digital circuits by integrating conducting polymers inside the leaves and xylem vascular tissue of Rosa floribunda. ${ }^{[2]}$ This breakthrough research opens doors for many applications since it demonstrated that the complex internal structure and physiology of plants could be used as a template and integral part of in-vivo manufactured electronic circuits. One of the most important biocompatible conducting polymers is poly(3,4-ethylenedioxythiophene) (PEDOT) due to its superior (bio) stability ${ }^{[3]}$ and high electronic ${ }^{[4]}$ and ionic conductivity. ${ }^{[5]}$ For $e$ Plants, ${ }^{[2]}$ a self-doped p-type PEDOT-derivative, PEDOT-S was used, ${ }^{[6]}$ which self-organized along the vascular tissue of the stem of the plant. In contrast to the PEDOT, PEDOT-S comprises covalently bound ionisable, functional groups (sulfonates) that can act as anions for self-doping. Self-doped polymers are more thermally stable than their "parent" or backbone conducting polymer due to potential crosslinking via the immobilized anions. ${ }^{[7]}$ This selfdoped crosslinked structure is also responsible for enhanced mechanical properties, ${ }^{[8]}$ and has 


\section{WILEY-VCH}

been leveraged, for example, in electronic control of cell detachment without the need of traditional biochemical methods. ${ }^{[9]}$

In the first $e$-Plants demonstration, electronic functionality could only be achieved in localized regions of the plant. ${ }^{[2]}$ Recently, we demonstrated self-organizing electronic functionality throughout the entire xylem vascular tissue without affecting or terminating the natural physiological processes of the plant. ${ }^{[10]}$ This was achieved by replacing PEDOT-S with the water soluble self-doped oligomer sodium salt of bis[3,4-ethylenedioxythiophene]3thiophene butyric acid (ETE-S) (Figure 1). ETE-S is effectively distributed and polymerized in the xylem vascular tissue with the plant acting as the template and catalyst for the polymerization reaction. The resulting xylem wires exhibit high conductivity, $10 \mathrm{~S} / \mathrm{cm}$, and specific capacitance of $20 \mathrm{~F} / \mathrm{g}$. The ETE-S based xylem wires have been used to manufacture a super capacitor in-vivo demonstrating a first practical application of the E-Plant technology where energy can be stored in a plant. Since ETE-S has shown great utility in templating electronic functionality inside living plants, the need for improvement of device performance and further functionalization of ETE-S motivates us to develop a detailed understanding of the nature of charge carriers and evolution of redox states in this material.

It has been well established for several decades, both theoretically and experimentally, that charge carriers in conducting polymers are quasiparticles such as polarons and bipolarons that are localized along the polymer backbone because of the strong electron-lattice interaction. ${ }^{[11]}$ The polarons represent localized charged states associated with a geometrical distortion of the polymer backbone. Formation of bipolarons takes place when the energy gain due to the rearrangement of the bond length exceeds the Coulomb energy related to the electron repulsion between two charges. Polarons and bipolarons are characterized by different electronic structures which, in turn, leads to distinct absorption and emission spectra. ${ }^{[12]}$ They can also be distinguished by means of electron paramagnetic resonance spectroscopy because bipolarons are spinless whereas polarons carry a magnetic moment. ${ }^{[13]}$ In addition, with two 


\section{WILEY-VCH}

charges on a chain, the character of geometrical distortion of the backbone is different for the case of a bipolaron and a polaron, which is manifested in distinct characteristics of the Raman spectra. ${ }^{[14]}$

Many studies have been reported addressing the nature of charge carriers in various polymers in the thiophene family using voltammetric, UV/Vis, and spectroelectrochemical techniques $^{[12 b, 15]}$ guided by theoretical calculations based on density-functional theory (DFT). ${ }^{[15 c, 15 f, 15 h-j, 16]}$ By now many of the observed features of the charged states and related optically-allowed transitions in these materials are well explained theoretically. However, there are still aspects that remain controversial and not fully understood: for example, a detailed origin of the charge carriers, i.e., polarons (radical cations) vs bipolarons (dications) or polaron pairs. ${ }^{[17]}$

In the present study, we report spectroelectrochemical characterization of the self-doped ETES conducting polymer combined with its cyclic voltammetry and EPR spectroscopy. The experimental results are analysed using time-dependent DFT (TDDFT), which provides a detailed description of the UV/Vis absorption spectra. Our combined theoretical and experimental study allows us to outline the nature of the redox states (polaron vs bipolaron) and discuss electronic characteristics of ETE-S thin films in both the neutral and oxidized states.

\section{Results and Discussion}

In this section we start with theoretical TDDFT calculations of optical absorption of reduced and oxidized ETE-S chains. We then report experimental characterization of ETE-S film by means of UV/Vis spectroelectrochemistry, cyclic voltammetry and electron paramagnetic resonance spectroscopy and analyse the experimental data on the basis of theoretical calculations. 


\section{WILEY-VCH}

\subsection{DFT and TDDFT calculations on a reduced and oxidized ETE-S chains.}

Range-separated hybrid functionals, such as $\omega \mathrm{B} 97 \mathrm{XD},{ }^{[18]}$ can accurately predict electronic structure and optical transitions in conducting polymers. ${ }^{[16 c, 16 \mathrm{~d}, 19]}$ The length of ETE-S oligomers after electropolymerization of the ETE-S is not precisely known, so we first investigated the absorption of single-chain oligomers with different chain length $n$ ranging from one to five ETE-S units, $1 \leq n \leq 5$, that is, three to fifteen thiophene units, since ETE-S is a thiophene trimer. The TDDFT computed spectrum and corresponding electronic transitions for fully reduced oligomers with different chain lengths $n$ are shown in Figure 2 . In the reduced state, each sulfonate group of oligomer is compensated by a sodium counter ion with the total charge of the system consisting of the oligomer and the counterion being zero. When the oligomer chain length increases from $n=1$ to $n=5$, the main optical transition is red-shifted from $350 \mathrm{~nm}$ to $487 \mathrm{~nm}$. However, as the chain length increases, the main peak of the absorption spectra converges around $490 \mathrm{~nm}$, with negligible difference between $n=4$ and $n=5$ (see Figure S1b). This result implies that four units are sufficient for accurate description of optical properties of long ETE-S polymers, which is consistent with corresponding behaviour reported previously for related oligomers. ${ }^{[16 c, 20]}$ Note that the height of the transition peaks is given by the oscillator strength $f$, which depends linearly on the chain length $n,{ }^{[21]}$ see Figure S1c. Therefore, when normalized to the chain length, the height of the main absorption peaks for different $n$ ETE-S would be practically the same for all $n$.

Figure $2 \mathrm{~b}$ shows electronic transitions, optimized ground state energies, and configuration interaction (CI) contribution to the main transitions for fully reduced oligomer of length $n=4$ calculated using TDDFT. As expected, the main optical transition is mainly due to the $\mathrm{HOMO} \rightarrow$ LUMO transition but other transitions are also involved. This conclusion holds for all studied chain lengths $n=1-5$. Parameters describing optical transitions for reduced oligomers of all lengths (i.e. the wavelength of the main optical transitions $\lambda$, the corresponding energies of the optical transitions $E_{\mathrm{opt}}=h c / \lambda$, the oscillator strength $f$, 


\section{WILEY-VCH}

molecular orbitals MOs and CI coefficients) are listed in Table S1; the main molecular orbitals involved in the ground state to the excited state transitions are visualized in Figure S2 and S7. We also calculated the HOMO-LUMO energy difference $\Delta E=E_{\mathrm{LUMO}}-E_{\mathrm{HOMO}}$ obtained from the DFT calculations, see Figure S1a and Table S2. It is noteworthy that $\Delta E$ is different from the corresponding energy of the optical transition $E_{\text {opt }}$ obtained by TDDFT (c.f. Figure S1a and S1b, and Table S1 and S2) because the former does not take into account electron-hole interaction, which can not be neglected for the system excited by the alternating electric field. ${ }^{[19 b],[21 \mathrm{a}]}$ As a result, $\Delta E$ is typically larger than the corresponding energy $E_{\text {opt. }}$.

With $n=4$ settled as a viable length for further study, we can begin to address the polaronic or bipolaronic nature of the charge carriers in the oxidized oligomers. On a fully reduced ETE-S oligomer chain we remove one counterion in order to form a polaronic state on the oligomer backbone while maintaining the electroneutrality of the system,

$(\text { ETE-S })^{-} \mathrm{Na}^{+} \rightarrow(\text { ETE-S })^{-}(\text {polaron })^{+}+\mathrm{Na}^{+}+e^{-}$

The polaron state is localized at the backbone (See Figure S3) and is characterized by two absorption bands at $715 \mathrm{~nm}$ and $1258 \mathrm{~nm}$ respectively (Figure 3a). Figure 3b shows electronic transitions, optimized ground state energies, and configuration interaction (CI) contributions to the main transitions for the polaronic state in the oligomer of the length $n=4$. The lowest energy absorption polaronic band at $1258 \mathrm{~nm}$ is due to transition from $\beta$ HOMO-1 $\rightarrow \beta$ LUMO and $\beta$ HOMO $\rightarrow \beta$ LUMO ( $\alpha$ and $\beta$ stand for different spin states). The high energy polaron band at $715 \mathrm{~nm}$ consists of two overlapping subbands at $707 \mathrm{~nm}$ and $737 \mathrm{~nm}$. The subband at $737 \mathrm{~nm}$ is mostly due to $\beta$ HOMO-2 $\rightarrow \beta$ LUMO and $\alpha$ HOMO-2 $\rightarrow \alpha$ LUMO transitions, while the subband at $707 \mathrm{~nm}$ is primarily caused by $\alpha$ HOMO-1 $\rightarrow \alpha$ LUMO and $\alpha \mathrm{HOMO} \rightarrow \alpha$ LUMO transitions. All contributing optical transitions are listed in 
Table S3; the main molecular orbitals involved in the ground state to the excited state transitions are visualized in Figure S3.

Again considering a fully reduced ETE-S oligomer chain we remove two counterions in order to form a bipolaronic state on the oligomer backbone while maintaining the electroneutrality of the system

$2(\text { ETE-S })^{-} 2 \mathrm{Na}^{+} \rightarrow 2(\text { ETE-S })^{-}(\text {bipolaron })^{2+}+2 \mathrm{Na}^{+}+2 e^{-}$.

In the bipolaron state, removing two $\mathrm{Na}^{+}$counterions leads to the alkyl sulfonate moiety bending toward the oligomer backbone (inset to Figure $3 \mathrm{c}$ ). As a result, a negatively charged sulfonate group enhances the localization of a bipolaronic state on the backbone as compared to the polaronic state (note that we remove counterions from neighbouring side chains, see Figure S4). The absorption spectra of the bipolaron state shown in Figure 3c exhibits a single band at $802 \mathrm{~nm}$. Figure $3 \mathrm{~d}$ shows three main contributions to the bipolaronic band at $802 \mathrm{~nm}$ including HOMO- $1 \rightarrow$ LUMO, HOMO-3 $\rightarrow$ LUMO, and HOMO-3 $\rightarrow$ LUMO+1 transitions. All the remaining transitions contributing to the bipolaronic band are listed in Table S2; Figure S4 visualizes the corresponding molecular orbitals.

It is noteworthy that the bipolaron absorption band as well as both absorption bands for the polaron state are considerably red-shifted compared to the case of reduced ETE-S oligomer. Indeed, the wavelength of the main transitions for fully reduced oligomer of length $n=4$ is $\lambda=483 \mathrm{~nm}$, whereas the corresponding wavelengths for the bipolaron band is $\lambda=802 \mathrm{~nm}$ and for the polaron band, $\lambda=1258 \mathrm{~nm}$ and $707 \mathrm{~nm}$, see Figure 2a and Table S3. This makes it possible to distinguish the presence of charge carriers in ETE-S oligomers. It should also be noted that a similar formation of a single absorption band for the bipolaron state and two absorption bands for the polaron state has been reported in previous studies of oligomers of the thiophene family and related polymers. ${ }^{[12 \mathrm{a}, 12 \mathrm{~b}, 15 \mathrm{a}, 15 \mathrm{~b}, 15 \mathrm{~h}]}$ 


\section{WILEY-VCH}

So far, we considered the absorption spectra of single chain oligomers. Molecular dynamic calculations show that ETE-S thin films comprise small crystallites composed of $\pi-\pi$ stacked oligomers. ${ }^{[10]}$ (Note that a similar stacked behaviour of oligomer chains was demonstrated in molecular dynamics calculations of PEDOT:TOS) ${ }^{[22]}$. In order to study the effect of polymer chain stacking, we performed DFT and TDDFT calculation on a system consisting of two $\pi-\pi$ stacked ETE-S oligomer chains in the reduced and bipolaronic states. Figure 4 shows the comparison of the corresponding absorption spectra for the single oligomer chain consisting of two ETE-S units and two $\pi-\pi$ stacked oligomer chains as illustrated in the insets.

Absorption spectra of reduced oligomers in the stacked configuration shows a single absorption band at $422 \mathrm{~nm}$, which exhibits a small blue shift $(15 \mathrm{~nm})$ due to the inter-chain interaction with respect to the case of a single oligomer chain, Figure 4a. For the bipolaron case the absorption spectra of two stacked ETE-S oligomer chains reveals one band with two subbands at 715 and $779 \mathrm{~nm}$ respectively, Figure 4b. (All contributing optical transitions and corresponding molecular orbitals are shown in Table S4 and Figure S5 and S6). Both subbands in absorption spectra of stacked ETE-S oligomer chain in the bipolaronic state are also blue shifted with respect to the case of a single oligomer (40 $\mathrm{nm}$ shift for the high energy subband and $104 \mathrm{~nm}$ for the low energy subband). The inter-chain interaction for the bipolaronic state has apparently a stronger effect as compared to the reduced state because the former exhibits a more pronounced blue shift. One would expect that realistic ETE-S thin films would contain both single and stacked chains. All of them would contribute to the absorption spectra, which would result in the broadening of the observed peaks.

\subsection{UV/Vis Spectroelectrochemistry and Electron paramagnetic resonance spectroscopy}

The doping state (or redox state) of conducting polymers can be changed electrochemically. In this case charge carriers are introduced to the backbone through a metal contact while at 


\section{WILEY-VCH}

the same time ions from an electrolyte enter the film in order to compensate the electronic charge. Spectroelectrochemistry represents a powerful technique for investigating the optical absorption of conducting polymer films while their doping state is changed electrochemically from the fully reduced (dedoped) to the fully oxidized state (doped).$^{[151,23]}$

Spectroelectrochemical data of an electropolymerized ETE-S film for various applied potentials in respect to $\mathrm{Ag} / \mathrm{AgCl}$ reference electrode are shown in Figure 5. At large enough negative applied potentials relative to the reference electrode, $-1 \mathrm{~V} \lesssim V \lesssim-0.3 \mathrm{~V}$, a single absorption band centred at $550 \mathrm{~nm}$ is observed. As the voltage increases toward $0 \mathrm{~V}$, this absorption band disappears, but a new absorption band centred at $800 \mathrm{~nm}$ develops. With further voltage increase in the interval $0 \mathrm{~V} \lesssim V \lesssim+0.7 \mathrm{~V}$, the intensity of the absorption peak at $800 \mathrm{~nm}$ decreases, and a broad tail starts to grow in the interval $\lambda \gtrsim 1000 \mathrm{~nm}$.

Spectroelecrochemical data are complemented by electron paramagnetic resonance (EPR) spectroscopy measurements that provide information on unpaired spins in the sample. ${ }^{[17 \mathrm{~b}, 24]}$ Figure 6 shows the EPR spectra of the electropolymerized ETE-S film at different doping states along with the corresponding spin count (i.e., the spin density of the measured spin-1/2 species). The observed trend in the density of spin-1/2 polarons - an increase until reaching a maximum followed by a decrease -is characteristic for bipolaron formation that pairs the spin- $1 / 2$ polarons to the spinless bipolarons. An increasing bipolaron formation is also indicated by the broadening of the EPR linewidth, as a result of increasing polaron interations with increasing polaron concentration, which in turn promotes formation of bipolarons. For the fully oxidized sample $(V=+0.7 \mathrm{~V})$ with the carrier density of about $33 \%$ per monomer, the number of the spin- $1 / 2$ charges (polarons) determined from the spin count amounts to $\sim 2 \%$ of the total carrier density, leaving $\sim 98 \%$ of the charge carriers to be spinless bipolarons. (In the above estimations we used the molecular weight of ETE-S $544 \mathrm{~g} / \mathrm{mol}$, the density $\rho=1 \mathrm{~g} / \mathrm{cm}^{3}$, and the dimensions of the film $1 \times 0.2 \times 10^{-5} \mathrm{~cm}^{3}$ ). Assuming that the charge density varies linearly between the fully oxidized $(V=+0.7 \mathrm{~V})$ and the fully reduced states $(V=-1 \mathrm{~V})$, we estimate that for the case of no 


\section{WILEY-VCH}

applied voltage $V=0 \mathrm{~V}$ the ratio between spin- and spinless charges (i.e., polarons:bipolarons) is $\sim 10 \%: 90 \%$. The above estimations are consistent with previous studies that report the dominance of spinless charge carriers in PEDOT and related conducting polymers from the thiophene family. ${ }^{[17 b, 24 b]}$ An estimation of the ratio between polarons and bipolarons becomes more difficult for the case of the reduced samples in the low charge density limit where cyclic voltammetry in the interval $-1 \mathrm{~V} \lesssim V \lesssim-0.7 \mathrm{~V}$ shows peaks related to redox reactions, presumably due to oxygen reduction. This makes it difficult to distinguish the contributions to the spin count due to polarons and due to possible molecular defects and trap states acting as a catalyst for such reactions. ${ }^{[25]}$ We should note that the g-value of the EPR signal obtained at $V$ $=-1 \mathrm{~V}$ is noticeably different from that from the other applied voltages, probably indicating that it arises from a different spin-1/2 species (e.g., traps) rather than polarons. This suggestion is supported by the observed broader EPR linewidth at $V=-1 \mathrm{~V}$ as compared with that at $-0.5 \mathrm{~V} \lesssim V \lesssim-0.2 \mathrm{~V}$, as the former would have the same or even narrower EPR linewidth if it originated from the same polarons but with a lower density.

\subsection{Discussion: nature of the charge carriers in ETE-S polymeric films}

Based on the computed electronic transitions and the EPR measurements we assign the peaks in the UV/Vis spectroelectrochemical data and suggest the nature of the charge carriers in the ETE-S film as follows. The reduced samples in the interval $-1 \mathrm{~V} \lesssim V \lesssim-0.7 \mathrm{~V}$ show a single pronounced peak at $550 \mathrm{~nm}$, which originates from the HOMO $\rightarrow$ LUMO transition in the neutral chains with no charge carriers. The absence of the polaronic peak in the spectra strongly suggests that the observed ERP signal is not related to polarons but instead is due to molecular defects or trap states as discussed above.

As the voltage is increased to $V \lesssim-0.3 \mathrm{~V}$ a single absorption band at $800 \mathrm{~nm}$ develops which is attributed to the bipolaronic states. The polarons are practically absent in this regime 


\section{WILEY-VCH}

because the absorption spectrum shows a negligible signal above $1200 \mathrm{~nm}$ (note that $1258 \mathrm{~nm}$ corresponds to the second polaronic band, see Figure 4a). The formation of charged carriers in the system in the given voltage range is also apparent from the cyclic voltammetry measurements, which exhibit a significant increase in the current (Figure S8). Thus, the formation of the absorption band at $800 \mathrm{~nm}$, along with the decrease of the peak at $550 \mathrm{~nm}$ in the oxidation process, indicates doping of the polymer film with bipolaron charge carriers and exchange of $\mathrm{Na}^{+}$counterions from the polymer film to the electrolyte.

With further increase of the applied voltage, the intensity of the bipolaronic peak $(800 \mathrm{~nm})$ increases; then it decreases and eventually saturates at $V \approx+0.7 \mathrm{~V}$. In this voltage range, some polaronic charged states are introduced, as evidenced by the spin signal. The spin count is maximal at $V \approx 0 \mathrm{~V}$ (Figure $6 \mathrm{~b}$ ), which corresponds to the polaron:bipolaron ratio $\sim 90 \%: 10 \%$ as discussed above. The spin count starts to decrease as the applied voltage increases above $0 \mathrm{~V}$, and for the fully oxidized sample bipolarons become fully dominant (polaron:bipolaron ratio $\sim 98 \%: 2 \%$ ). It is noteworthy that the decrease of the spin count for $V \gtrsim 0 \mathrm{~V}$ is accompanied by the increase of the EPR line broadening (Figure 6b). The change of the character of the EPR broadening is typically attributed to the change of the character of charge carriers, which is consistent with the present case when the polaron:bipolaron ratio changes from $\sim 10 \%: 90 /$ at $0 \mathrm{~V}$ to $\sim 2 \%: 98 \%$ at $+0.7 \mathrm{~V}$.

Finally, there is a broad tail in the absorption spectra $(\lambda \gtrsim 1000 \mathrm{~nm})$ that starts to develop for $V \gtrsim 0 \mathrm{~V}$ and becomes dominant in the fully oxidized state. Nominally, this tail matches the wavelength region corresponding to the lowest energy polaronic absorption band (Figure 3a). This match is however coincidental as the origin of the tail is not related to the polaronic band. Indeed, according to the TDDFT calculations the intensity of the absorption in this wavelength region would not exceed the intensity of the highest energy polaronic band, which is apparently not the case. Moreover, the tail becomes the most pronounced for the fully oxidized state of ETE-S film which, according to the EPR signal, exhibits a negligible 
fraction of polarons. We attribute the origin of the tail to the drastic changes of the band structure and to the strong decrease of the band gap when a polymeric film undergoes oxidation. It has been theoretically demonstrated recently that when the charge carrier concentration increases, the band structure of a realistic polymer thin film exhibits significant transformation, showing a strong decrease of the band gap as compared to the neutral case accompanied by the increase of the density of states in the band gap. ${ }^{[26]}$ The origin of this behavior is related to the effect of randomly located counterions giving rise to mid-gap states. We thus attribute the observed tail to the HOMO $\rightarrow$ LUMO transition in the highly oxidized polymeric film, noting that the LUMO states in this case represent the bipolaronic states ${ }^{[26]}$. Apparently, the observed tail cannot be reproduced by the DFT calculation for a single chain because for its accurate description, one has to account for the complex morphology of crystallites including the effects of counterions.

\section{Conclusion}

The recently synthetized water-soluble self-doped ETE-S oligomer shows great potential as a material for $e$-Plant (and other bioelectronics) applications as it can polymerize in vivo, without application of external stimuli forming conducting wires and electrodes for energy storage in the xylem vascular tissue of plants. ${ }^{[10]}$ A detailed understanding of the nature of the charge carriers in this material is thus essential for developing device applications and improving material performance. In the present study, we investigate how the nature of charge carriers evolves when electrochemically-polymerized ETE-S films undergo a reversible transition from reduced to electrically conductive oxidized states. This has been done using spectroelecrochemical measurements combined with electron paramagnetic resonance spectrometry, cyclic voltammetry, and supported by DFT and time-dependent DFT calculations. 


\section{WILEY-VCH}

Spectroelecrochemical measurements was proven to be a powerful technique to investigate absorbance changes of conducting polymer film under electrochemical doping. We show that the absorption spectra of ETE-S films in contact with electrolyte strongly depend on the applied bias, and that changes in absorption spectra are associated with varying doping level in ETE-S film with the applied potential.

The reduced samples show a single pronounced peak at $550 \mathrm{~nm}$, which is related to the HOMO $\rightarrow$ LUMO transition in the neutral chains with no charge carriers. As the oxidation level is increased a single absorption band at $800 \mathrm{~nm}$ develops, which is attributed to spinless bipolaronic states. With further increase of the voltage, some polaronic charged states possessing a magnetic moment are introduced in the system; the spin count is maximal at $0 \mathrm{~V}$, which corresponds to a polaron:bipolaron ratio $\sim 10 \%: 90 \%$. The spin count decreases as the applied voltage increases above $0 \mathrm{~V}$, and for the fully oxidized sample bipolarons become fully dominant (polaron:bipolaron ratio $\sim 2 \%: 98 \%$ ). At the same time, a broad tail in the absorption spectra starts to develop in the region $\lambda \gtrsim 1000 \mathrm{~nm}$, and becomes dominant in the fully oxidized state. We attribute the origin of the tail to the drastic changes of the band structure leading to the strong decrease of the band gap when a polymeric film undergoes oxidation. This significantly deeper understanding of ETE-S will enable future $e$-Plant and other bioelectronics applications to take advantage of more tunable and optimized devices, paving the way for even deeper integration of organic electronics with biological systems.

\section{Experimental Section}

DFT and TDFT calculations: Quantum mechanical calculations were carried out using time dependent density functional calculations, implemented in the GAUSSIAN 09 software package. ${ }^{[27]}$ Range-separated hybrid functional $\omega \mathrm{B} 97 \mathrm{XD}^{[18]}$, which includes $22 \%$ HartreeFock (HF) exchange at short-range and $100 \%$ HF exchange at long range, with additional 


\section{WILEY-VCH}

Grimme's D2 $2^{[28]}$ dispersion correction were utilized in the calculation. Initially, ground state geometries were optimized with $3-21 \mathrm{~g}$ basis set in gas phase. All the conformations obtained at $\omega \mathrm{B} 97 \mathrm{XD} / 3-21 \mathrm{~g}$ level were again fully reoptimized using $6-31 \mathrm{~g}(\mathrm{~d})$ basis set in water solvent. The water solvent effects are taken into account within implicit solvation polarizable continuum model $(\mathrm{PCM})^{[29]}$ as implemented in the GAUSSIAN package. Symmetry constraints were not taken into consideration during geometric optimization. Singlet state calculations were carried out using spin-restricted DFT (RDFT), corresponding to the bipolaron states, and spin-unrestricted DFT (UDFT), corresponding to the polaron states. To get the absorption spectrum, excitation energies were calculated using the time dependent density functional theory method (TDDFT) at $\omega \mathrm{B} 97 \mathrm{XD} / 6-31 \mathrm{~g}(\mathrm{~d})$ level within PCM. Visualization of results was achieved through GaussView 5.0.9 $9^{[30]}$, GaussSum 3.0 $0^{[31]}$ and Gabedit $^{[32]}$.

ETE-S electropolymerization and cyclic voltammetry characterization: We used a standard three electrode electrochemical set up with a Pt sheet as the counter electrode and saturated $\mathrm{Ag} / \mathrm{AgCl}$ as the reference electrode. All measurements were performed using a $\mu$ Autolab potentiostat with Nova 1.6 software.

ETE-S was electropolymerized on ITO-coated glass to form a polymer film. The electrolyte consisted of $1 \mathrm{mg} / \mathrm{ml}$ ETE-S in $0.01 \mathrm{M} \mathrm{NaCl}(\mathrm{aq})$. The film was deposited by cyclic voltammetry ( 5 scans, $0.5--0.5 \mathrm{~V}, 50 \mathrm{mV} / \mathrm{s}$ ) followed by applying constant current of $250 \mu \mathrm{A}$ for $150 \mathrm{sec}$. This resulted in a film of about $80 \mathrm{~nm}$ thickness. To record the $\mathrm{CV}$, the film was rinsed in DI water and left to dry. For $\mathrm{CV}$, we used $0.01 \mathrm{M} \mathrm{NaCl}\left(\mathrm{aq}\right.$ ) purged with $\mathrm{N}_{2}$ for two hours before the acquisition of the data.

ETE-S spectroelectrochemistry: UV/Vis spectroelectrochemistry was performed in a quartz cuvette that contained the three electrode setup in $0.01 \mathrm{M} \mathrm{NaCl}(\mathrm{aq})$. In this case, the working electrode consisted of the electropolymerized ETE-S film on ITO coated glass. The UV/Vis 


\section{WILEY-VCH}

spectra (300-1250 nm, step $1 \mathrm{~nm})$ were taken using a PerkinElmer Lambda 900 spectrometer while the film was biased at a certain potential.

EPR spectroscopy: EPR measurements were performed on an X-band Bruker Elexsys E500 spectrometer, in the dark and at room temperature. All EPR spectra were obtained with 2 Gs magnetic field modulation at a modulation frequency of $100 \mathrm{kHz}$. To avoid the strong perturbation of the electrolyte to the EPR cavity, the doping state of the ETE-S film was changed electrochemically outside of the EPR cavity. In this case we used a two electrode setup comprising of the electropolymerized film and a $\mathrm{Ag} / \mathrm{AgCl}$ counter electrode in $0.01 \mathrm{M}$ LiCl4 in acetonitrile. . The EPR measurements were performed within 5 minutes after the electrochemical treatments, which should provide a reliable measure of the spin density as the EPR signal only decreased by $5 \%$ after an hour. The background EPR signal was measured from the reference substrate sample without the ETE-S film, and was subtracted from all EPR spectra. The spin counts were calculated using the Xenon software, taking into account the Qvalues of the EPR cavity for every EPR spectrum. The spin densities were then obtained by normalizing the spin counts by the sample volume.

To take into account a slight difference in the microwave frequencies for different measurement runs and to facilitate a direct comparison between the EPR spectra, Fig.6(a) was plotted with EPR signal intensity as a function of g-factor instead of magnetic field following the relationship $g=\frac{h f}{\mu_{B} B}$. Here, $f$ denotes the microwave frequency, $h$ the Planck constant, $m_{B}$ the Bohr magneton, and $B$ the external magnetic field.

\section{Supporting Information}

Supporting Information is available from the Wiley Online Library or from the author.

\section{Acknowledgements}




\section{WILEY-VCH}

This work was supported by multiple funding sources: The Swedish Energy Agency (grant 38332-1), the Norrköpings fond för Forskning och Utveckling, Carl Tryggers Stiftelse för Vetenskaplig Forskning (CTS: 13 527), CeNano (Linköping University), the Knut and Alice Wallenberg foundation (Tail of the Sun), the Swedish Foundation for Strategic Research (SSF), and the Advanced Functional Material SFO-center at Linköping University (Faculty Grant SFO-Mat-LiU 2009-00971). E.S. is also supported by a Marie Sklodowska Curie Individual Fellowship (MSCA-IF-EF-ST, Trans-Plant, 702641). The computations were performed on resources provided by the Swedish National Infrastructure for Computing (SNIC) at NSC.

Received: ((will be filled in by the editorial staff))

Revised: ((will be filled in by the editorial staff)) Published online: ((will be filled in by the editorial staff))

\section{References}

[1] D. T. Simon, E. O. Gabrielsson, K. Tybrandt, M. Berggren, Chemical Reviews 2016, 116, 13009.

[2] E. Stavrinidou, R. Gabrielsson, E. Gomez, X. Crispin, O. Nilsson, D. T. Simon, M. Berggren, Science Advances 2015, 1.

[3] H. Yamato, M. Ohwa, W. Wernet, Journal of Electroanalytical Chemistry 1995, 397, 163.

[4] L. Groenendaal, F. Jonas, D. Freitag, H. Pielartzik, J. R. Reynolds, Advanced Materials 2000, 12, 481.

[5] E. Stavrinidou, P. Leleux, H. Rajaona, D. Khodagholy, J. Rivnay, M. Lindau, S. Sanaur, G. G. Malliaras, Advanced Materials 2013, 25, 4488.

[6] R. H. Karlsson, A. Herland, M. Hamedi, J. A. Wigenius, A. Åslund, X. Liu, M. Fahlman, O. Inganäs, P. Konradsson, Chemistry of Materials 2009, 21, 1815.

[7] I. Yu, B. A. Deore, C. L. Recksiedler, T. C. Corkery, A. S. Abd-El-Aziz, M. S. Freund, Macromolecules 2005, 38, 10022.

[8] B. A. Deore, I. Yu, P. M. Aguiar, C. Recksiedler, S. Kroeker, M. S. Freund, Chemistry of Materials 2005, 17, 3803.

[9] K. M. Persson, R. Karlsson, K. Svennersten, S. Löffler, E. W. H. Jager, A. RichterDahlfors, P. Konradsson, M. Berggren, Advanced Materials 2011, 23, 4403. 


\section{WILEY-VCH}

[10] E. Stavrinidou, R. Gabrielsson, K. P. R. Nilsson, S. K. Singh, J. F. Franco-Gonzalez, A. V. Volkov, M. P. Jonsson, A. Grimoldi, M. Elgland, I. V. Zozoulenko, D. T. Simon, M. Berggren, Proceedings of the National Academy of Sciences 2017, DOI: 10.1073/pnas.1616456114.

[11] W. R. Salaneck, R. H. Friend, J. L. Brédas, Physics Reports 1999, 319, 231.

[12] a) J. Cornil, D. Beljonne, J. L. Brédas, The Journal of Chemical Physics 1995, 103, 842 ; b) J. A. E. H. van Haare, E. E. Havinga, J. L. J. van Dongen, R. A. J. Janssen, J. Cornil, J.-L. Brédas, Chemistry - A European Journal 1998, 4, 1509; c) J. Hwang, D. B. Tanner, I. Schwendeman, J. R. Reynolds, Physical Review B 2003, 67, 115205.

[13] Y. Harima, T. Eguchi, K. Yamashita, K. Kojima, M. Shiotani, Synthetic Metals 1999, $105,121$.

[14] S. R. González, Y. Ie, Y. Aso, J. T. López Navarrete, J. Casado, Journal of the American Chemical Society 2011, 133, 16350.

[15] a) J. J. Apperloo, R. A. J. Janssen, P. R. L. Malenfant, L. Groenendaal, J. M. J. Fréchet, Journal of the American Chemical Society 2000, 122, 7042; b) P. J. Brown, H. Sirringhaus, M. Harrison, M. Shkunov, R. H. Friend, Physical Review B 2001, 63, 125204; c) J. J. Apperloo, L. B. Groenendaal, H. Verheyen, M. Jayakannan, R. A. J. Janssen, A. Dkhissi, D. Beljonne, R. Lazzaroni, J.-L. Brédas, Chemistry - A European Journal 2002, 8, 2384; d) Y. Y. Deng, H. Sirringhaus, Physical Review B 2005, 72, 045207; e) N. Fomina, S. E. Bradforth, T. E. Hogen-Esch, Macromolecules 2009, 42, 6440; f) J. Aragó, P. M. Viruela, E. Ortí, R. Malavé Osuna, B. Vercelli, G. Zotti, V. Hernández, J. T. López Navarrete, J. T. Henssler, A. J. Matzger, Y. Suzuki, S. Yamaguchi, Chemistry - A European Journal 2010, 16, 5481; g) J. M. Szarko, B. S. Rolczynski, J. Guo, Y. Liang, F. He, M. W. Mara, L. Yu, L. X. Chen, The Journal of Physical Chemistry B 2010, 114, 14505; h) L. Zaikowski, P. Kaur, C. Gelfond, E. Selvaggio, S. Asaoka, Q. Wu, H.-C. Chen, N. Takeda, A. R. Cook, A. Yang, J. Rosanelli, J. R. Miller, Journal of the American Chemical Society 2012, 134, 10852; i) J. Aragó, R. Ponce Ortiz, B. Nieto-Ortega, V. Hernández, J. Casado, A. Facchetti, T. J. Marks, P. M. Viruela, E. Ortí, J. T. López Navarrete, ChemPhysChem 2012, 13, 168; j) J. C. Earles, H. van der Salm, P. Wagner, D. L. Officer, K. C. Gordon, Journal of Molecular Structure 2013, 1047, 80; k) B. Lu, S. Zhen, S. Zhang, J. Xu, G. Zhao, Polymer Chemistry 2014, 5, 4896; 1) S. Ming, S. Zhen, K. Lin, L. Zhao, J. Xu, B. Lu, ACS Applied Materials \& Interfaces 2015, 7, 11089; m) Z. Xu, M. Wang, W. Fan, J. Zhao, H. Wang, Electrochimica Acta 2015, 160, 271; n) S. Guan, A. S. Elmezayyen, F. Zhang, J. Zheng, C. Xu, Journal of Materials Chemistry C 2016, 4, 4584; o) J. Shi, X. Zhu, P. Xu, M. Zhu, Y. Guo, Y. He, Z. Hu, I. Murtaza, H. Yu, L. Yan, O. Goto, H. Meng, Macromolecular Rapid Communications 2016, 37, 1344; p) L. Kaiwen, C. Shuai, L. Baoyang, X. Jingkun, SCIENCE CHINA Chemistry 2017, 60, 38.

[16] a) E. Zojer, J. Cornil, G. Leising, J. L. Brédas, Physical Review B 1999, 59, 7957; b) F. Alkan, U. Salzner, The Journal of Physical Chemistry A 2008, 112, 6053; c) U. Salzner, Wiley Interdisciplinary Reviews: Computational Molecular Science 2014, 4, 601; d) H. Sun, J. Autschbach, Journal of Chemical Theory and Computation 2014, 10, 1035.

[17] a) N. Zamoshchik, M. Bendikov, Advanced Functional Materials 2008, 18, 3377; b) H. Tanaka, S. Nishio, H. Ito, S.-i. Kuroda, Applied Physics Letters 2015, 107, 243302; c) V. M. Geskin, J.-L. Brédas, ChemPhysChem 2003, 4, 498.

[18] J.-D. Chai, M. Head-Gordon, Physical Chemistry Chemical Physics 2008, 10, 6615.

[19] a) U. Salzner, Journal of Chemical Theory and Computation 2007, 3, 1143; b) L. Kronik, T. Stein, S. Refaely-Abramson, R. Baer, Journal of Chemical Theory and Computation 2012, 8, 1515; c) U. Salzner, The Journal of Physical Chemistry A 2008, 112, 5458. 
[20] a) U. Salzner, A. Aydin, Journal of Chemical Theory and Computation 2011, 7, 2568;

b) R. Telesca, H. Bolink, S. Yunoki, G. Hadziioannou, P. T. Van Duijnen, J. G. Snijders, H. T. Jonkman, G. A. Sawatzky, Physical Review B 2001, 63, 155112.

[21] a) S. Tretiak, K. Igumenshchev, V. Chernyak, Physical Review B 2005, 71, 033201; b)

L. Zheng, N. F. Polizzi, A. R. Dave, A. Migliore, D. N. Beratan, The Journal of Physical Chemistry A 2016, 120, 1933.

[22] J. F. Franco-Gonzalez, I. V. Zozoulenko, The Journal of Physical Chemistry B 2017, DOI: $10.1021 /$ acs.jpcb.7b01510.

[23] a) A. Cihaner, F. Alg1, Advanced Functional Materials 2008, 18, 3583; b) G. E. Gunbas, A. Durmus, L. Toppare, Advanced Functional Materials 2008, 18, 2026; c) S. Jeeva, O. Lukoyanova, A. Karas, A. Dadvand, F. Rosei, D. F. Perepichka, Advanced Functional Materials 2010, 20, 1661; d) A. P. Bonifas, R. L. McCreery, Analytical Chemistry 2012, 84, 2459; e) G. Sonmez, H. Meng, F. Wudl, Chemistry of Materials 2003, 15, 4923; f) M. Vilas-Boas, C. Freire, B. de Castro, P. A. Christensen, A. R. Hillman, Inorganic Chemistry 1997, 36, 4919; g) A. M. Fraind, J. D. Tovar, The Journal of Physical Chemistry B 2010, 114, 3104.

[24] a) A. Zykwinska, W. Domagala, M. Lapkowski, Electrochemistry Communications 2003, 5, 603; b) O. Bubnova, Z. U. Khan, H. Wang, S. Braun, D. R. Evans, M.

Fabretto, P. Hojati-Talemi, D. Dagnelund, J.-B. Arlin, Y. H. Geerts, S. Desbief, D. W. Breiby, J. W. Andreasen, R. Lazzaroni, W. M. Chen, I. Zozoulenko, M. Fahlman, P. J. Murphy, M. Berggren, X. Crispin, Nat Mater 2014, 13, 190.

[25] L. Zhang, Z. Xia, The Journal of Physical Chemistry C 2011, 115, 11170.

[26] W. A. Muñoz, S. K. Singh, J. F. Franco-Gonzalez, M. Linares, X. Crispin, I. V. Zozoulenko, Physical Review B 2016, 94, 205202.

[27] M. J. Frisch, G. W. Trucks, H. B. Schlegel, G. E. Scuseria, M. A. Robb, J. R. Cheeseman, G. Scalmani, V. Barone, B. Mennucci, G. A. Petersson, H. Nakatsuji, M. Caricato, X. Li, H. P. Hratchian, A. F. Izmaylov, J. Bloino, G. Zheng, J. L. Sonnenberg, M. Hada, M. Ehara, K. Toyota, R. Fukuda, J. Hasegawa, M. Ishida, T. Nakajima, Y. Honda, O. Kitao, H. Nakai, T. Vreven, J. A. Montgomery, J. E. Peralta, F. Ogliaro, M. Bearpark, J. J. Heyd, E. Brothers, K. N. Kudin, V. N. Staroverov, R. Kobayashi, J. Normand, K. Raghavachari, A. Rendell, J. C. Burant, S. S. Iyengar, J. Tomasi, M. Cossi, N. Rega, J. M. Millam, M. Klene, J. E. Knox, J. B. Cross, V. Bakken, C. Adamo, J. Jaramillo, R. Gomperts, R. E. Stratmann, O. Yazyev, A. J. Austin, R. Cammi, C. Pomelli, J. W. Ochterski, R. L. Martin, K. Morokuma, V. G. Zakrzewski, G. A. Voth, P. Salvador, J. J. Dannenberg, S. Dapprich, A. D. Daniels, Farkas, J. B. Foresman, J. V. Ortiz, J. Cioslowski, D. J. Fox, Gaussian 09, Revision B.01, Wallingford CT 2009.

[28] S. Grimme, Journal of Computational Chemistry 2006, 27, 1787.

[29] J. Tomasi, B. Mennucci, R. Cammi, Chemical Reviews 2005, 105, 2999.

[30] K. T. Dennington R, Millam J GaussView, Version 5.0.8.: Semichem Inc., Shawnee Mission KS. 2009.

[31] N. M. O'Boyle, A. L. Tenderholt, K. M. Langner, Journal of Computational Chemistry 2008, 29, 839.

[32] A.-R. Allouche, Journal of Computational Chemistry 2011, 32, 174. 


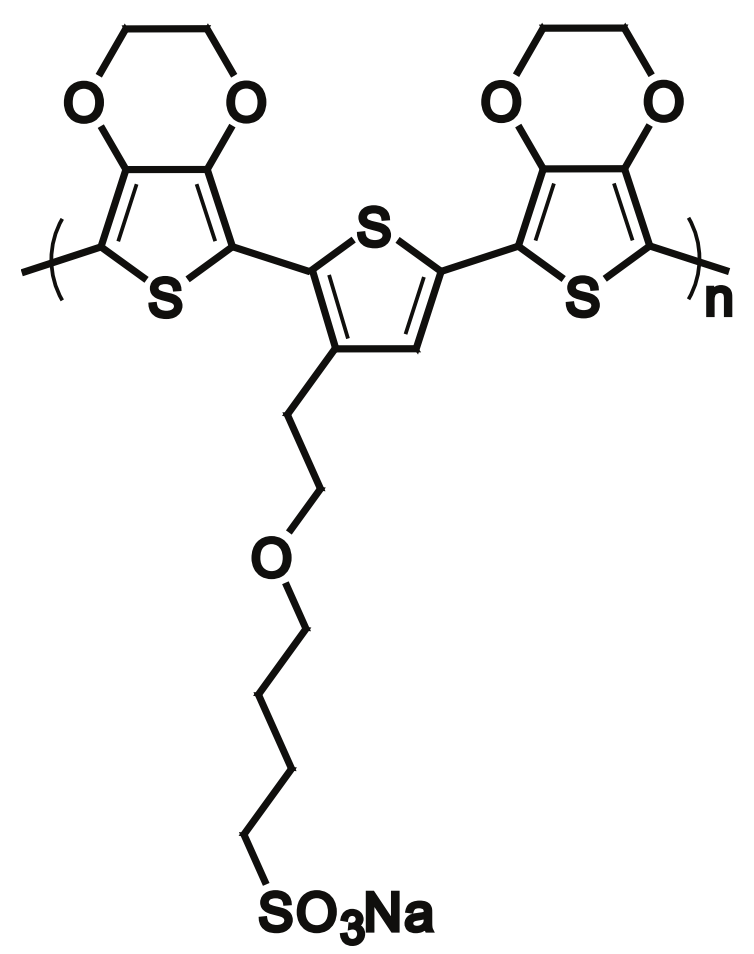

Figure 1. Chemical structure of the self-doped conducting polymer ETE-S in the reduced state when the sulfonate group of the oligomer is compensated by a $\mathrm{Na}^{+}$. The tri-thiophene shown represents one monomer unit of ETE-S. 


\section{WILEY-VCH}

a)

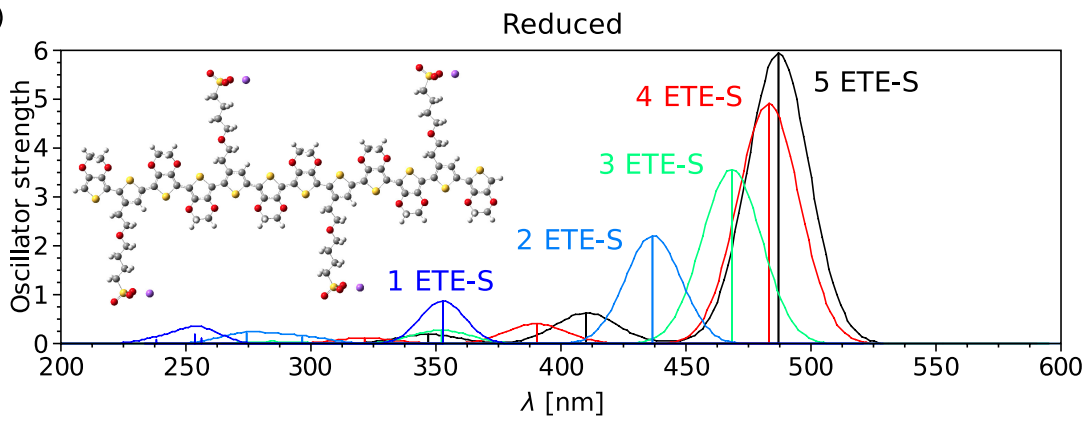

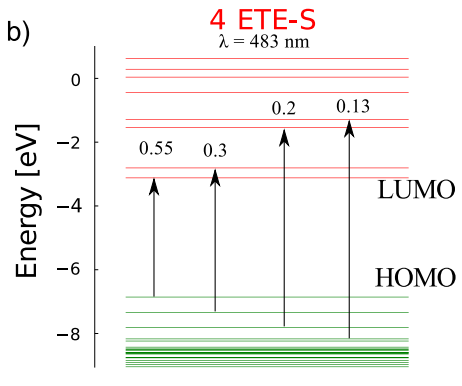

Figure 2. (a) UV/Vis spectra of ETE-S oligomers with different chain lengths $n=1-5$ in a fully reduced electronic state (1 ETE-S through 5 ETE-S respectively). Inset shows 4 ETE-S oligomer; here and hereafter $\mathrm{C}$ atoms are shown in grey, $\mathrm{O}$ in red, $\mathrm{S}$ in yellow, and $\mathrm{Na}^{+}$ counterions in purple. (b) Ground state energy structure for molecular orbitals involved in the main optical absorption peak for fully reduced 4 ETE-S. Numbers indicate configuration interaction (CI) contributions of different transitions to the absorption peak. 
a)

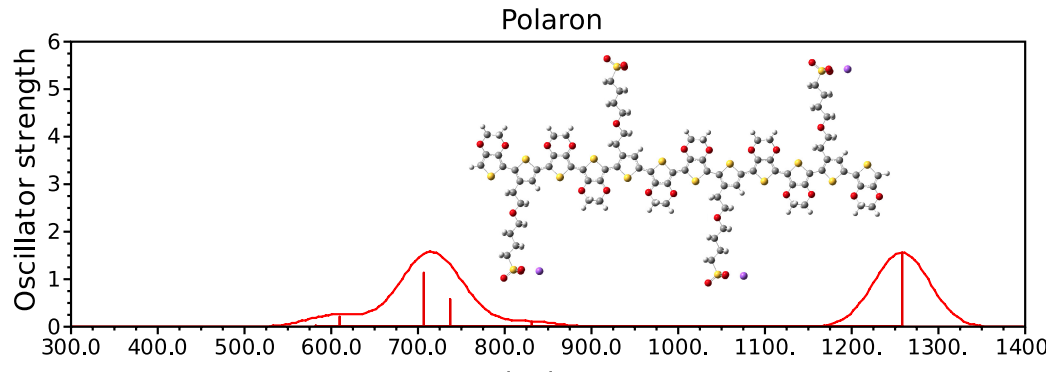

c)

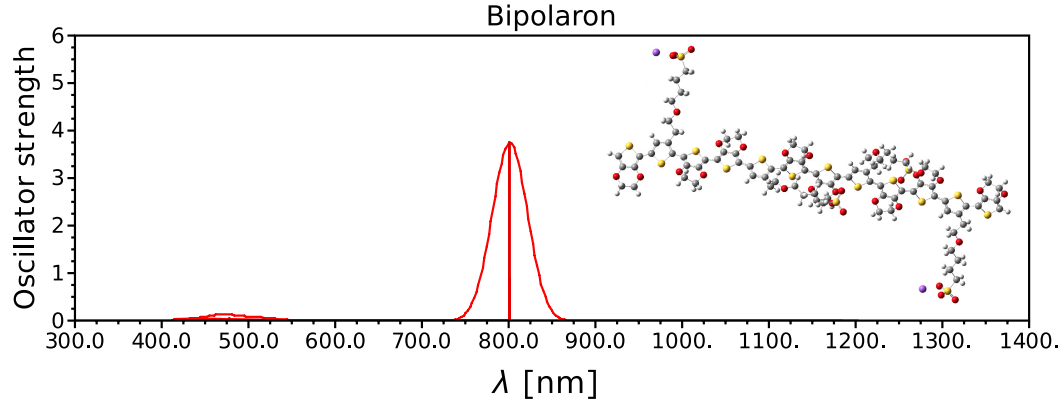

b)

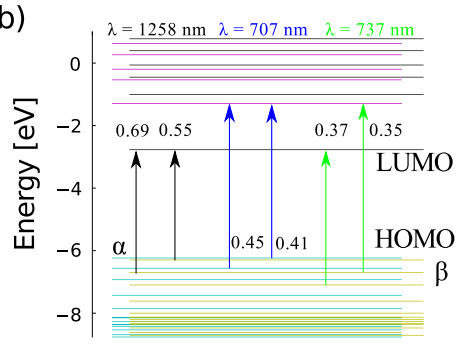

d)

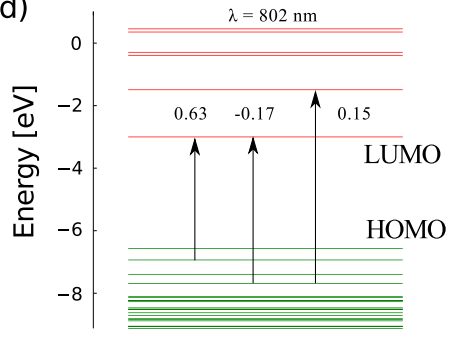

Figure 3. (a) UV/Vis spectra of 4 ETE-S oligomers in (a) polaron and (c) bipolaron states. These states are created by removing one and two $\mathrm{Na}^{+}$counterions from respectively (a) the second and (c) the second and third alkyl sulfonate moieties as shown in insets. Ground state energy structure for molecular orbitals involved in the main optical absorption peak for 4 ETE-S oligomers in (b) polaron and (d) bipolaron states. Numbers indicate configuration interaction (CI) contributions of different transitions to the absorption peak. 


\section{WILEY-VCH}

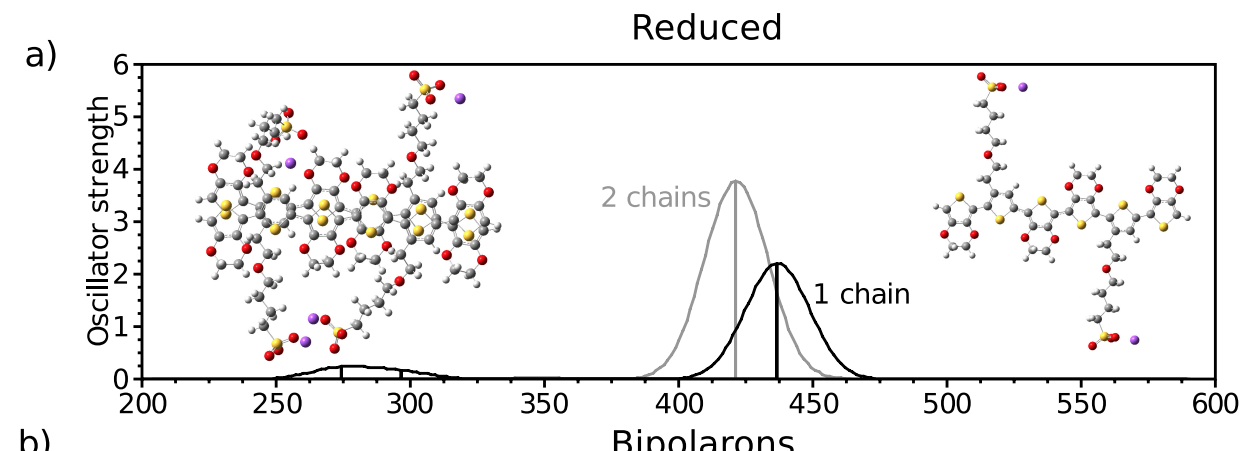

b)

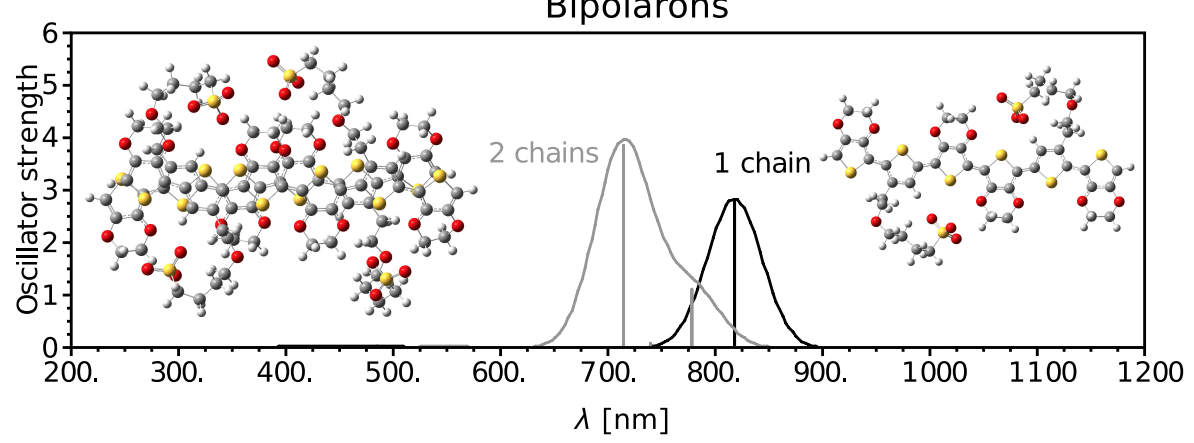

Figure 4. Effect of oligomer $\pi-\pi$ stacking on UV/Vis spectra for (a) reduced and (b) bipolaronic states. (In (b) each chain contains one bipolaron state). 


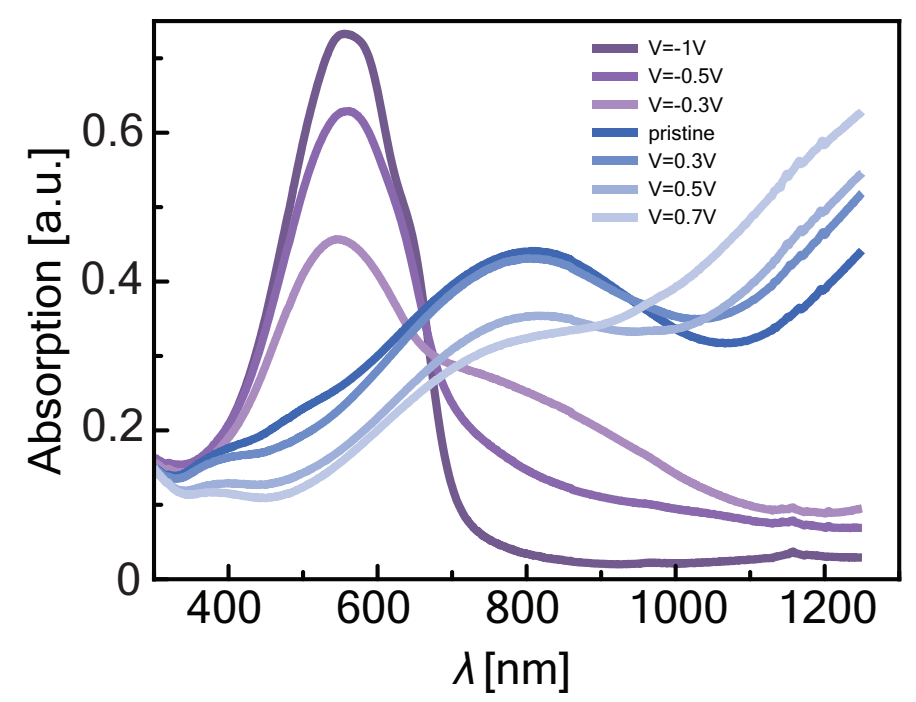

Figure 5. Spectroelectrochemical data of ETE-S film in $0.01 \mathrm{M} \mathrm{NaCl(aq)} \mathrm{solution.}$ 
WILEY-VCH
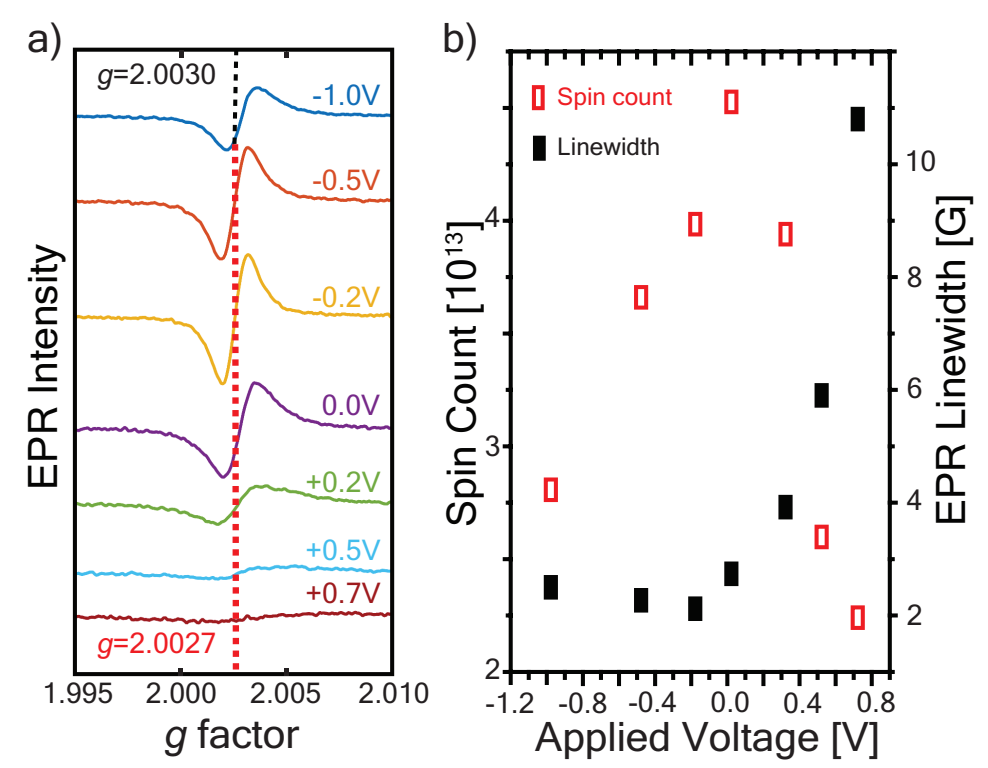

Figure 6. (a) EPR spectra of the ETE-S film after electrochemical doping at different applied potentials, shifted vertically for clarity. (b) Spin count and EPR linewidth for the spectra shown in (a). 


\section{WILEY-VCH}

\section{The table of contents}

The nature of charge carriers in water soluble self-doped conducting polymer ETE-S

during a reversible transition from reduced to electrically conductive oxidized state was

elucidated through spectroelecrochemical measurements combined with electron

paramagnetic resonance spectrometry, cyclic voltammetry, and supported by DFT and time-

dependent DFT calculations.

Keywords: self-doped conducting polymers; sodium salt of bis[3,4-

ethylenedioxythiophene]3thiophene butyric acid (ETE-S); spectroelectrochemistry; polarons, bipolarons; density-functional theory

Anton V. Volkov, Sandeep Kumar Singh, Eleni Stavrinidou, Roger Gabrielsson, Juan F.

Franco-Gonzalez, Alex Cruce, Weimin M. Chen, Daniel T. Simon, Magnus Berggren and Igor V. Zozoulenko*

Spectroelectrochemistry and nature of charge carriers in self-doped conducting polymer

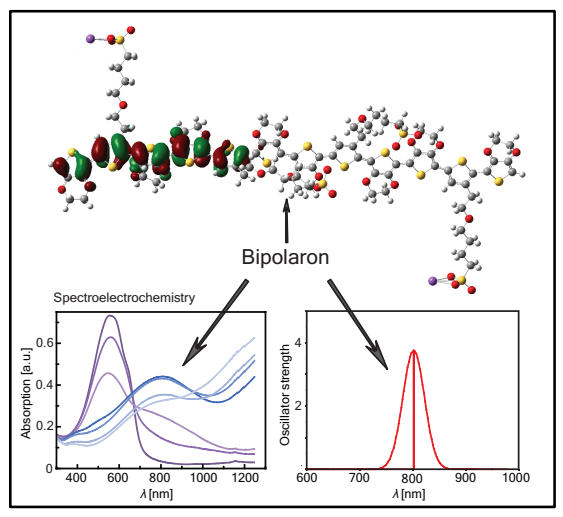

ToC figure ((Please choose one size: $55 \mathrm{~mm}$ broad $\times 50 \mathrm{~mm}$ high or $110 \mathrm{~mm}$ broad $\times 20 \mathrm{~mm}$ high. Please do not use any other dimensions)) 


\section{WILEY-VCH}

((Supporting Information can be included here using this template))

Copyright WILEY-VCH Verlag GmbH \& Co. KGaA, 69469 Weinheim, Germany, 2016.

\section{Supporting Information}

\section{Spectroelectrochemistry and nature of charge carriers in self-doped conducting polymer}

Anton V. Volkov, Sandeep Kumar Singh, Eleni Stavrinidou, Roger Gabrielsson, Juan F. Franco-Gonzalez, Alex Cruce, Weimin M. Chen, Daniel T. Simon, Magnus Berggren and Igor V. Zozoulenko*

Table S1. Evaluated parameters $\left(\lambda, E_{\mathrm{opt}}=h c / \lambda\right.$, the oscillator strength $f$, molecular orbitals MOs and CI coefficients) involved in the main optical transitions of reduced ETE-S oligomers with different chain length

\begin{tabular}{|c|c|c|c|c|c|}
\hline $\begin{array}{l}\text { Oligomer } \\
\text { Configuration }\end{array}$ & $\lambda(\mathrm{nm})$ & $\mathrm{E}_{\mathrm{opt}}(\mathrm{eV})$ & $f$ & MOs & $\mathrm{Cl}$ coefficient \\
\hline 1 ETE-S & 353 & 3,51339 & 0.8750 & $\begin{aligned} \mathrm{H} & \rightarrow \mathrm{L} \\
\mathrm{H}-4 & \rightarrow \mathrm{L}+2\end{aligned}$ & $\begin{array}{c}0.69031 \\
-0.11544\end{array}$ \\
\hline 2 ETE-S & 437 & 2,83632 & 2. 2230 & $\begin{aligned} \mathrm{H}-1 & \rightarrow \mathrm{L}+1 \\
\mathrm{H} & \rightarrow \mathrm{L}\end{aligned}$ & $\begin{array}{l}0.22230 \\
0.64953\end{array}$ \\
\hline 3 ETE-S & 469 & 2,64556 & 3.5750 & $\begin{array}{c}\mathrm{H} \rightarrow \mathrm{L} \\
\mathrm{H}-1 \rightarrow \mathrm{L}+1 \\
\mathrm{H}-2 \rightarrow \mathrm{L}+2\end{array}$ & $\begin{array}{c}0.59836 \\
-0.28182 \\
0.15635\end{array}$ \\
\hline 4 ETE-S & 483 & 2,56537 & 4.9262 & $\begin{array}{c}\mathrm{H} \rightarrow \mathrm{L} \\
\mathrm{H}-1 \rightarrow \mathrm{L}+1 \\
\mathrm{H}-2 \rightarrow \mathrm{L}+2 \\
\mathrm{H}-3 \rightarrow \mathrm{L}+3\end{array}$ & $\begin{array}{c}0.55127 \\
--0.30637 \\
-0.19897 \\
0.12629\end{array}$ \\
\hline 5 ETE-S & 487 & 2,54467 & 5.9565 & $\begin{array}{c}\mathrm{H} \rightarrow \mathrm{L} \\
\mathrm{H}-1 \rightarrow \mathrm{L}+1 \\
\mathrm{H}-2 \rightarrow \mathrm{L}+2 \\
\mathrm{H}-3 \rightarrow \mathrm{L}+3 \\
\mathrm{H}-4 \rightarrow \mathrm{L}+4\end{array}$ & $\begin{array}{c}0.52083 \\
-0.30432 \\
-0.21200 \\
-0.15240 \\
-0.10471\end{array}$ \\
\hline
\end{tabular}




\section{WILEY-VCH}

Table S2. The HOMO and LUMO energy levels, $E_{\text {HOMO }}$ and $E_{\text {LUMO, }}$ the HOMO-LUMO energy difference $\Delta E=E_{\mathrm{LUMO}}-E_{\mathrm{HOMO}}$ and the corresponding wavelength $\lambda_{\Delta E}=h c / \Delta E$ for $n$ ETE-S oligomers with different chain length $n$.

\begin{tabular}{lcccc}
\hline $\begin{array}{l}\text { Oligomer } \\
\text { Configuration }\end{array}$ & $\mathrm{E}_{\mathrm{HOMO}}$ & $\mathrm{E}_{\mathrm{LUMO}}$ & $\Delta E(\mathrm{eV})$ & $\lambda_{\Delta E}(\mathrm{~nm})$ \\
\hline 1 ETE-S & -6.796315772 & 0.139866524 & 6.936182296 & 178.7438604 \\
\hline 2 ETE-S & -6.30270123 & -0.381775746 & 5.920925484 & 209.3929409 \\
\hline 3 ETE-S & -6.152494379 & -0.536064305 & 5.616430075 & 220.7452035 \\
\hline 4 ETE-S & -6.087459167 & -0.603276428 & 5.484182738 & 226.068324 \\
\hline 5 ETE-S & -6.081200548 & -0.628855131 & 5.452345417 & 227.3883816 \\
\hline
\end{tabular}


Table S3. Evaluated parameters ( $\lambda$, the oscillator strength $f$, molecular orbitals MOs and CI coefficients) involved in the main optical transitions of reduced, polaron and bipolaron electronic states of oligomers with $n=4$ ETE-S units

\begin{tabular}{|c|c|c|c|c|}
\hline $\begin{array}{l}\text { Electronic } \\
\text { state }\end{array}$ & $\lambda(\mathrm{nm})$ & $f$ & MOs & $\mathrm{Cl}$ coefficient \\
\hline Reduced & 483 & 4.9262 & $\begin{aligned} \mathrm{H} & \rightarrow \mathrm{L} \\
\mathrm{H}-1 & \rightarrow \mathrm{L}+1 \\
\mathrm{H}-2 & \rightarrow \mathrm{L}+2 \\
\mathrm{H}-3 & \rightarrow \mathrm{L}+3\end{aligned}$ & $\begin{array}{c}0.55127 \\
--0.30637 \\
-0.19897 \\
0.12629\end{array}$ \\
\hline \multirow[t]{3}{*}{ Polaron } & 1258 & 2. 2230 & $\begin{array}{ll}\beta & \mathrm{H}-4 \rightarrow \mathrm{L} \\
\beta & \mathrm{H}-3 \rightarrow \mathrm{L} \\
\beta & \mathrm{H}-2 \rightarrow \mathrm{L}+1 \\
\beta & \mathrm{H}-1 \rightarrow \mathrm{L} \\
\beta & \mathrm{H} \rightarrow \mathrm{L} \\
\beta & \mathrm{H} \rightarrow \mathrm{L}+1 \\
\beta & \mathrm{H}-1 \leftarrow \mathrm{L}\end{array}$ & $\begin{array}{c}-0.15571 \\
-0.31012 \\
-0.19781 \\
0.68608 \\
0.54850 \\
0.10554 \\
0.12912\end{array}$ \\
\hline & 737 & 0.5839 & $\begin{array}{ll}\alpha & H-4 \rightarrow L \\
\alpha & H-3 \rightarrow L+3 \\
\alpha & H-3 \rightarrow L \\
\alpha & H-3 \rightarrow L+2 \\
\alpha & H-2 \rightarrow L \\
\alpha & H-1 \rightarrow L+1 \\
\alpha & H-1 \rightarrow L+3 \\
\alpha & H \rightarrow L \\
\alpha & H \rightarrow L+2 \\
\beta & H-7 \rightarrow L \\
\beta & H-3 \rightarrow L \\
\beta & H-2 \rightarrow L \\
\beta & H-2 \rightarrow L+2 \\
\beta & H-2 \rightarrow L+4 \\
\beta & H-1 \rightarrow L+1 \\
\beta & H-1 \rightarrow L+3 \\
\beta & H \rightarrow L \\
\beta & H \rightarrow L+1 \\
\beta & H \rightarrow L+2\end{array}$ & $\begin{array}{c}-0.10136 \\
0.13477 \\
0.12611 \\
-0.19816 \\
0.34827 \\
-0.30965 \\
-0.12512 \\
0.22542 \\
-0.20893 \\
0.16069 \\
0.16083 \\
0.36731 \\
0.12155 \\
0.11706 \\
-0.27669 \\
-0.17535 \\
-0.18235 \\
-0.12408 \\
0.19957\end{array}$ \\
\hline & 707 & 1.1615 & $\begin{array}{ll}\alpha & H-4 \rightarrow L+1 \\
\alpha & H-3 \rightarrow L \\
\alpha & H-3 \rightarrow L+2 \\
\alpha & H-2 \rightarrow L \\
\alpha & H-2 \rightarrow L+1 \\
\alpha & H-1 \rightarrow L \\
\alpha & H-1 \rightarrow L+2 \\
\alpha & H \rightarrow L \\
\alpha & H \rightarrow L+2 \\
\alpha & H \rightarrow L+3 \\
\beta & H-2 \rightarrow L \\
\beta & H-2 \rightarrow L+2 \\
\beta & H-1 \rightarrow L+1 \\
\beta & H-1 \rightarrow L+2 \\
\beta & H \rightarrow L+2 \\
\beta & H \rightarrow L+3\end{array}$ & $\begin{array}{c}0.11900 \\
0.21908 \\
0.11280 \\
-0.19441 \\
-0.25291 \\
0.45152 \\
-0.10648 \\
0.40567 \\
0.25416 \\
-0.13452 \\
-0.13954 \\
-0.10240 \\
0.14827 \\
0.15211 \\
-0.18964 \\
-0.22325\end{array}$ \\
\hline Bipolaron & 802 & 3.7571 & $\begin{array}{c}\mathrm{H}-3 \rightarrow \mathrm{L} \\
\mathrm{H}-3 \rightarrow \mathrm{L}+1 \\
\mathrm{H}-1 \rightarrow \mathrm{L}\end{array}$ & $\begin{array}{l}0.16689 \\
0.14502 \\
0.63645\end{array}$ \\
\hline
\end{tabular}




\section{WILEY-VCH}

Table S4. Evaluated parameters ( $\lambda$, the oscillator strength $f$, molecular orbitals MOs and CI coefficients) involved in the main optical transitions of ETE-S system consisting of single oligomer with $n=2$ ETE-S units and molecular cluster of two stacked oligomers with $\mathrm{n}=2 \mathrm{ETE}-\mathrm{S}$ units in fully reduced and bipolaronic states

\begin{tabular}{|c|c|c|c|c|}
\hline Configuration & $\lambda(\mathrm{nm})$ & $f$ & MOs & $\mathrm{Cl}$ coefficient \\
\hline \multirow[t]{2}{*}{$\begin{array}{l}2 \text { ETE-S } 2 \text { stacked } \\
\text { chain in Bipolaron } \\
\text { state }\end{array}$} & 779 & 1.1369 & $\begin{array}{c}\mathrm{H}-1 \rightarrow \mathrm{L} \\
\mathrm{H} \rightarrow \mathrm{L}\end{array}$ & $\begin{array}{c}0.63856 \\
-0.23539\end{array}$ \\
\hline & 714 & 3. 8785 & $\begin{array}{c}\mathrm{H}-2 \rightarrow \mathrm{L} \\
\mathrm{H}-2 \rightarrow \mathrm{L}+2 \\
\mathrm{H}-1 \rightarrow \mathrm{L} \\
\mathrm{H} \rightarrow \mathrm{L}+1\end{array}$ & $\begin{array}{c}-0.12083 \\
-0.10644 \\
0.21036 \\
0.63503\end{array}$ \\
\hline $\begin{array}{l}2 \text { ETE-S } 1 \text { chain in } \\
\text { Bipolaron state }\end{array}$ & 819 & 2.8463 & $\begin{aligned} \mathrm{H}-1 & \rightarrow \mathrm{L}+1 \\
\mathrm{H} & \rightarrow \mathrm{L}\end{aligned}$ & $\begin{array}{l}0.15919 \\
0.67880\end{array}$ \\
\hline $\begin{array}{l}2 \text { ETE-S } 2 \text { stacked } \\
\text { chain reduced }\end{array}$ & 421 & 3.7906 & $\begin{array}{c}\mathrm{H}-3 \rightarrow \mathrm{L}+2 \\
\mathrm{H}-2 \rightarrow \mathrm{L}+3 \\
\mathrm{H}-1 \rightarrow \mathrm{L} \\
\mathrm{H} \rightarrow \mathrm{L}+1\end{array}$ & $\begin{array}{c}0.16291 \\
0.14146 \\
0.49921 \\
-0.41046\end{array}$ \\
\hline $\begin{array}{l}2 \text { ETE-S } 1 \text { chain } \\
\text { reduced }\end{array}$ & 437 & 2.223 & $\begin{aligned} \mathrm{H}-1 & \rightarrow \mathrm{L}+1 \\
\mathrm{H} & \rightarrow \mathrm{L}\end{aligned}$ & $\begin{array}{l}0.22230 \\
0.64953\end{array}$ \\
\hline
\end{tabular}




\section{WILEY-VCH}

a)

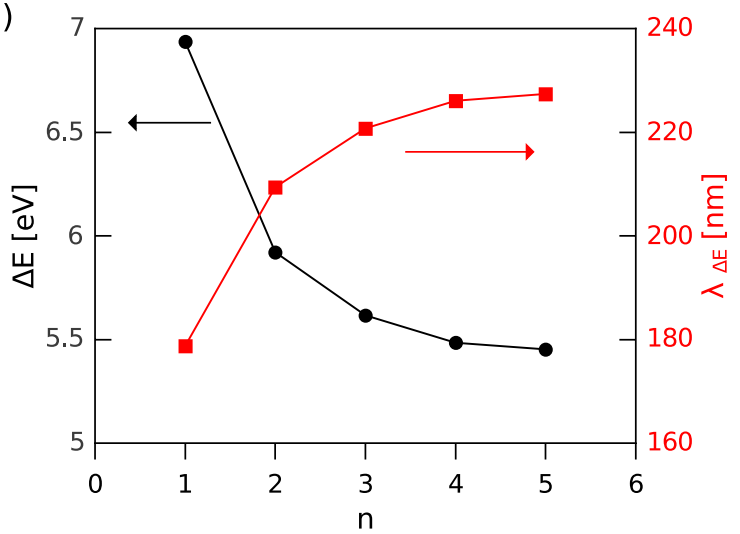

b)

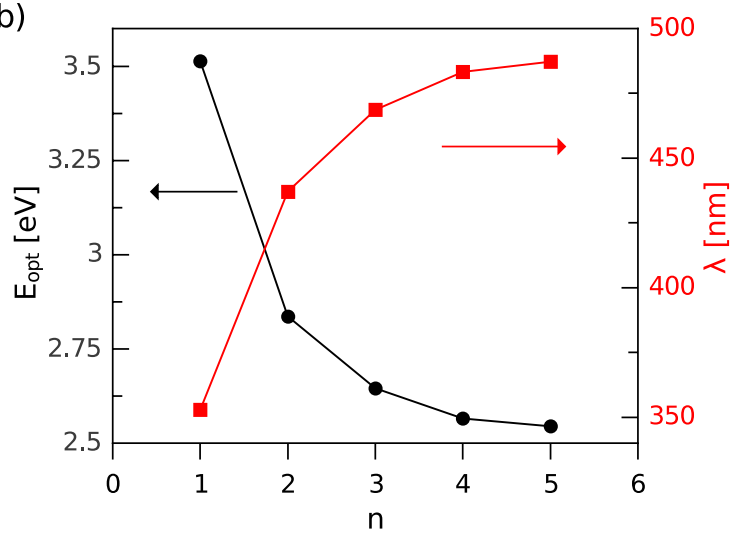

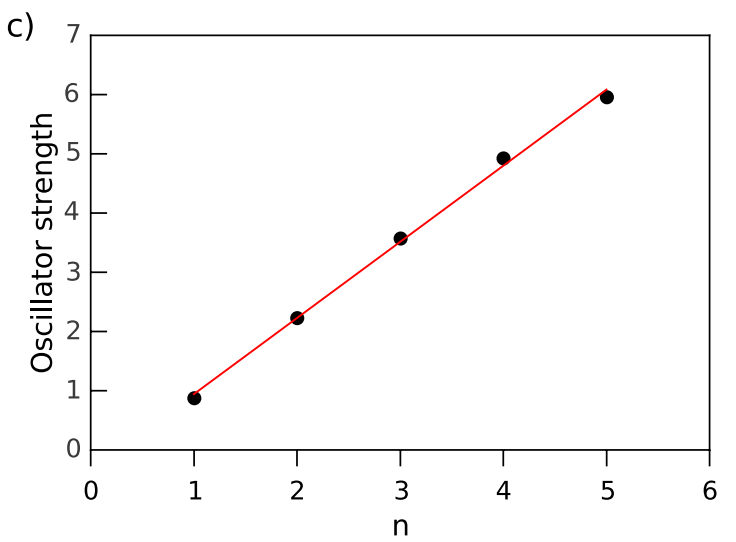

Figure S1: (a) The energy difference $\Delta E=E_{\mathrm{LUMO}}-E_{\mathrm{HOMO}}$ obtained from the DFT calculations and the corresponding wavelength as $\lambda_{\Delta E}=h c / \Delta E$ as a function of the number of ETE-S units $n$ in the chain. (b) The wavelength $\lambda$ corresponding to the main optical transitions in Fig. 2 and the corresponding optical gap $E_{\mathrm{opt}}=h c \lambda$ (here $h$ is Planck constant and $c$ speed of light) calculated from the TDDFT as a function of the number of ETE-S units $n$ in the chain.

(c) The oscillator strength for the main optical transitions in Figure 2 obtained from TDDFT as a function of the number of ETE-S units $n$ in the chain. 

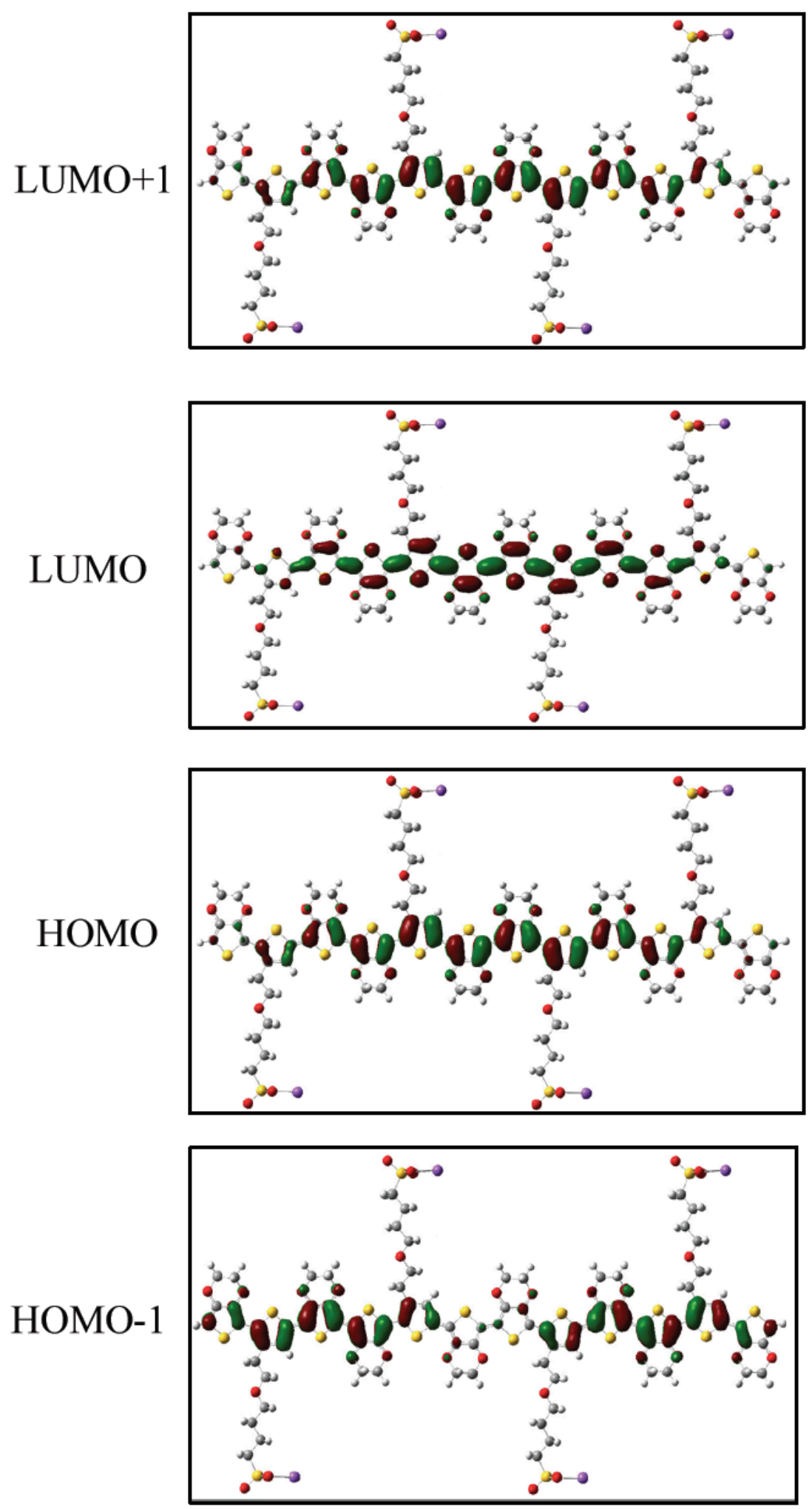

Figure S2. Main molecular orbitals involved (see Table S1) in the ground state to the excited state transitions for reduced oligomer with $n=4$ ETE-S units. 


\section{WILEY-VCH}
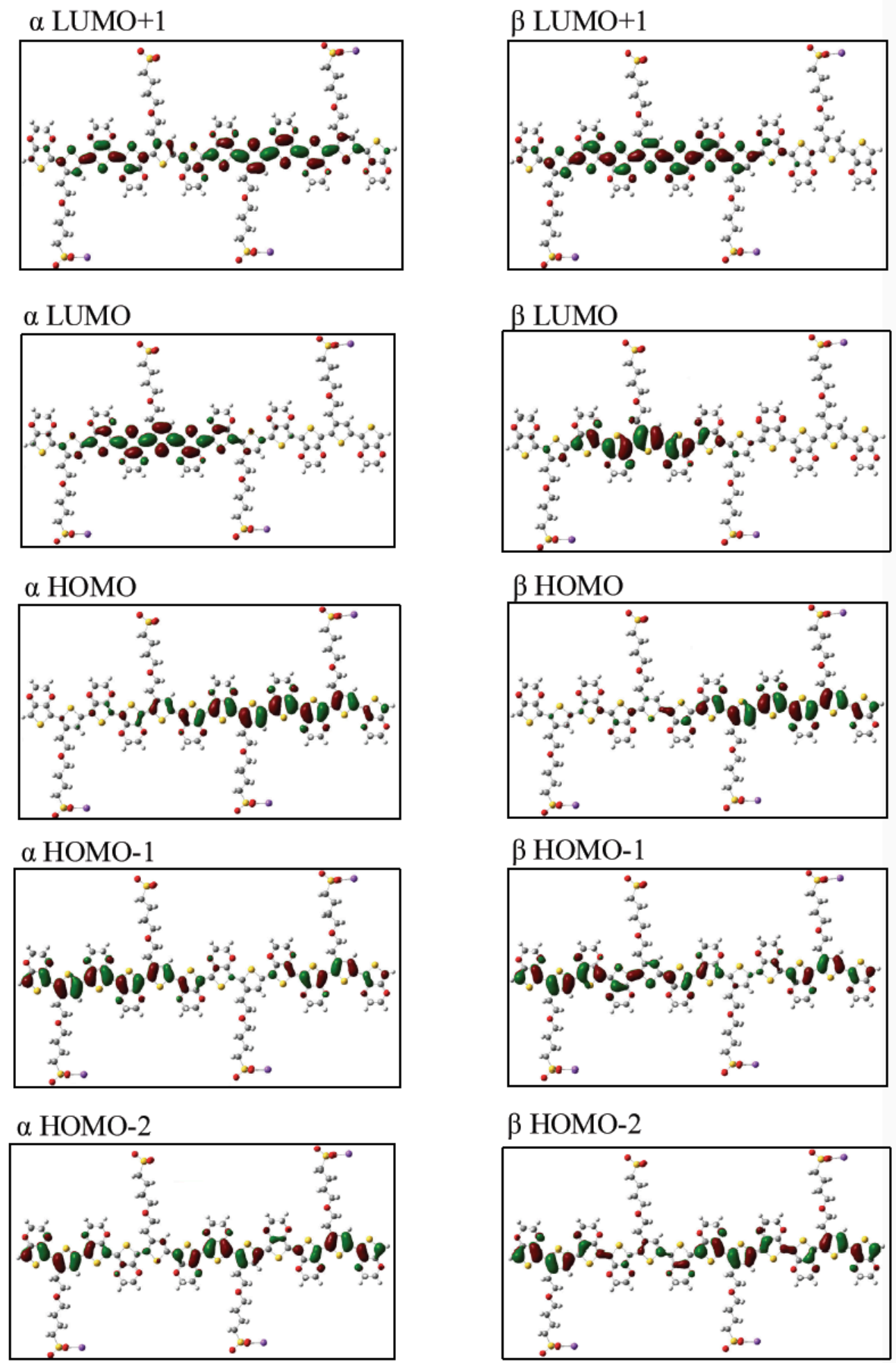

Figure S3. Main molecular orbitals involved (see Table S3) in the ground state to the excited state transitions for oligomer in polaron state with $n=4$ ETE-S units. $\alpha$ and $\beta$ refer to different spin states. 

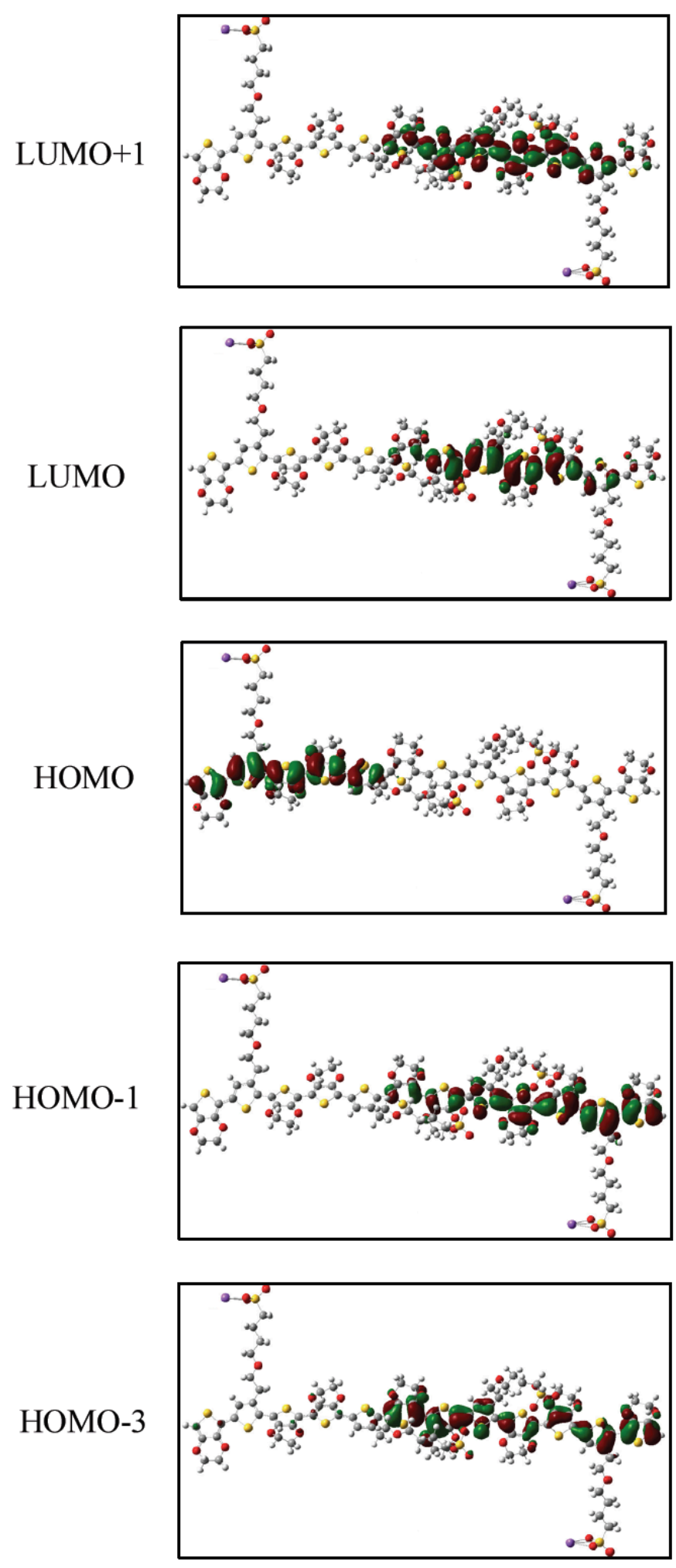

Figure S4. Main molecular orbitals involved (see Table S3) in the ground state to the excited states transitions for oligomer in bipolaron state with $n=4$ ETE-S units. 


\section{WILEY-VCH}
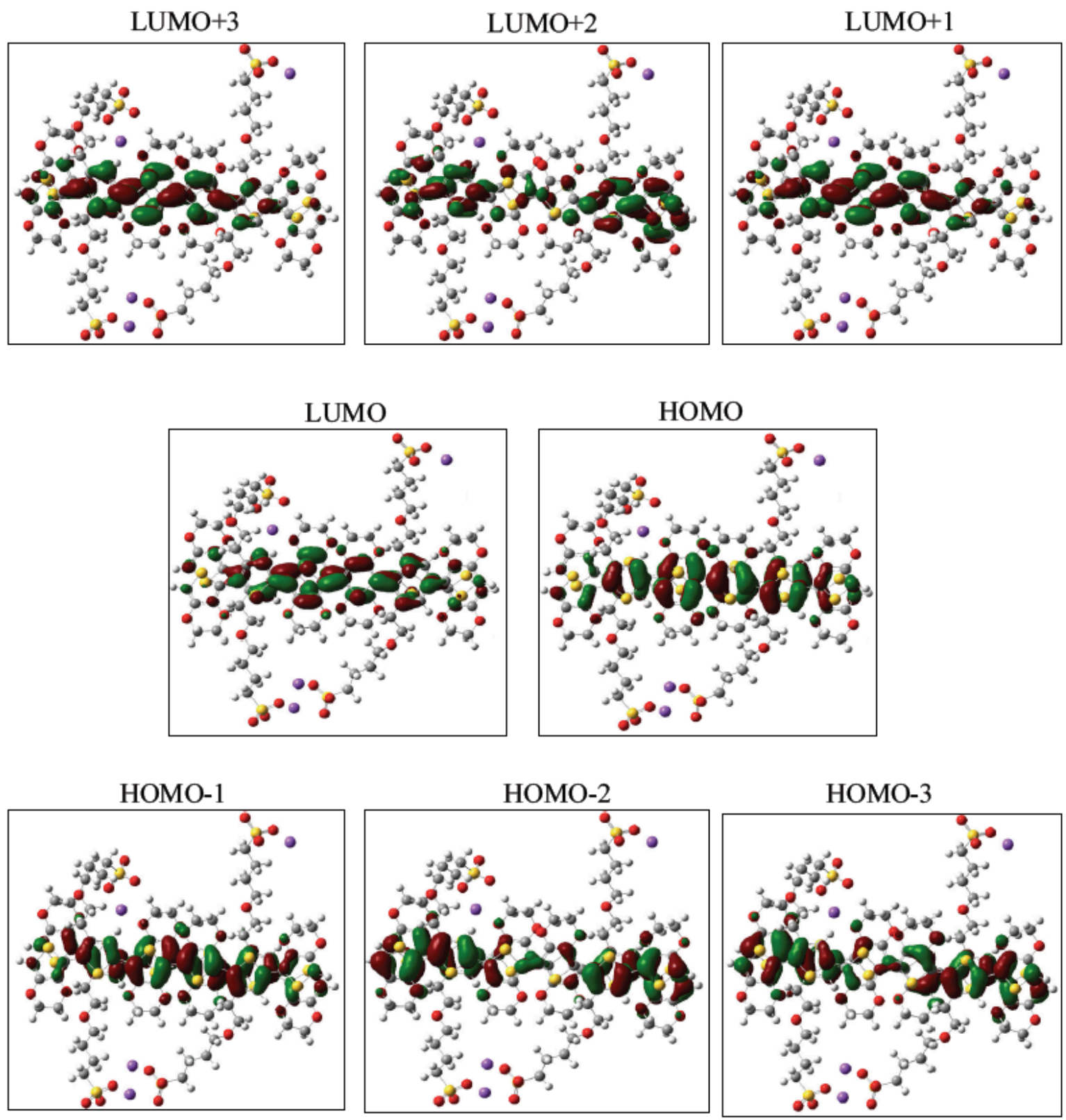

Figure S5. Main molecular orbitals involved (see Table S4) in the ground state to the excited states transitions for 2 stacked reduced oligomer with $n=2$ ETE-S units. 


\section{WILEY-VCH}

$\mathrm{LUMO}+3$

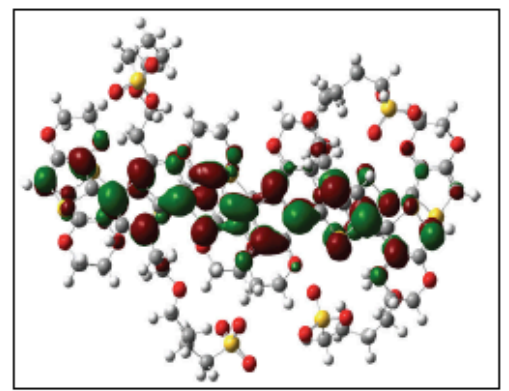

$\mathrm{LUMO}+2$

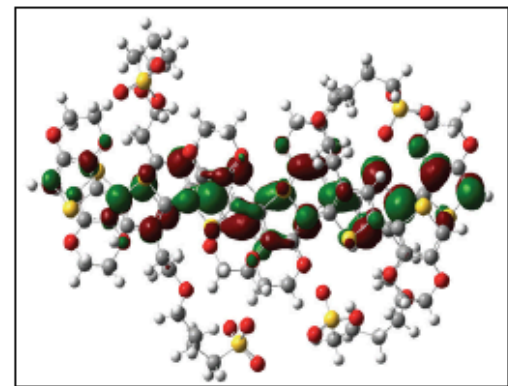

LUMO+1

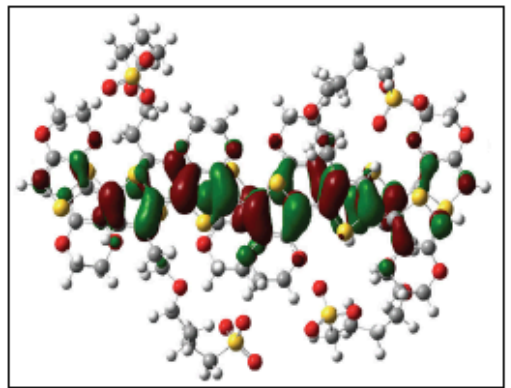

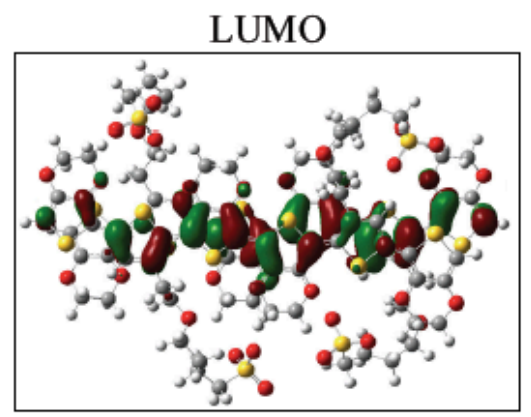

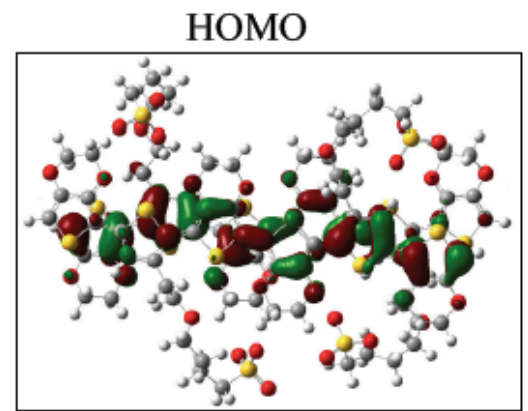

HOMO-1

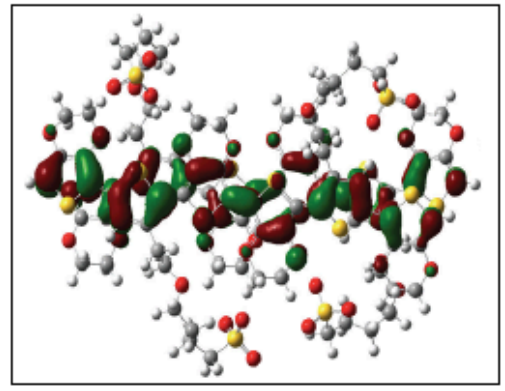

HOMO-2

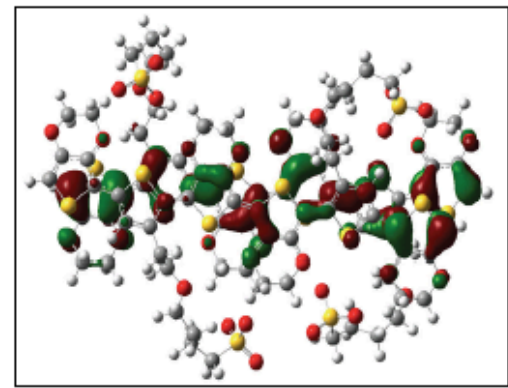

HOMO-3

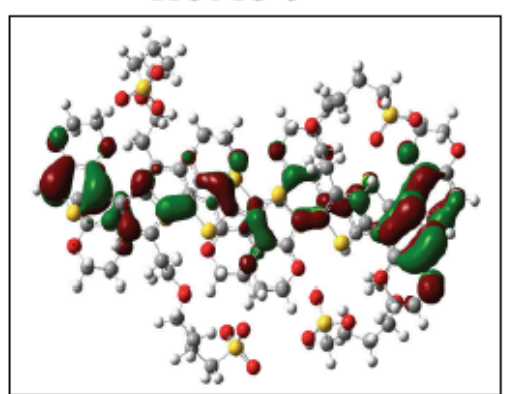

Figure S6. Main molecular orbitals involved (see Table S4) in the ground state to the excited states transitions for 2 stacked oligomer with $n=2$ ETE-S units in bipolaron state. 


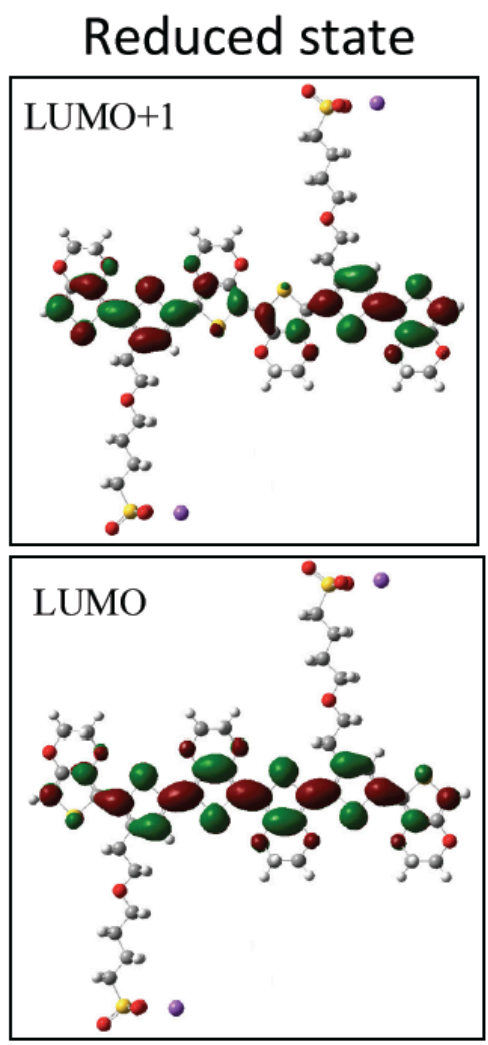

\section{Bipolaron state}
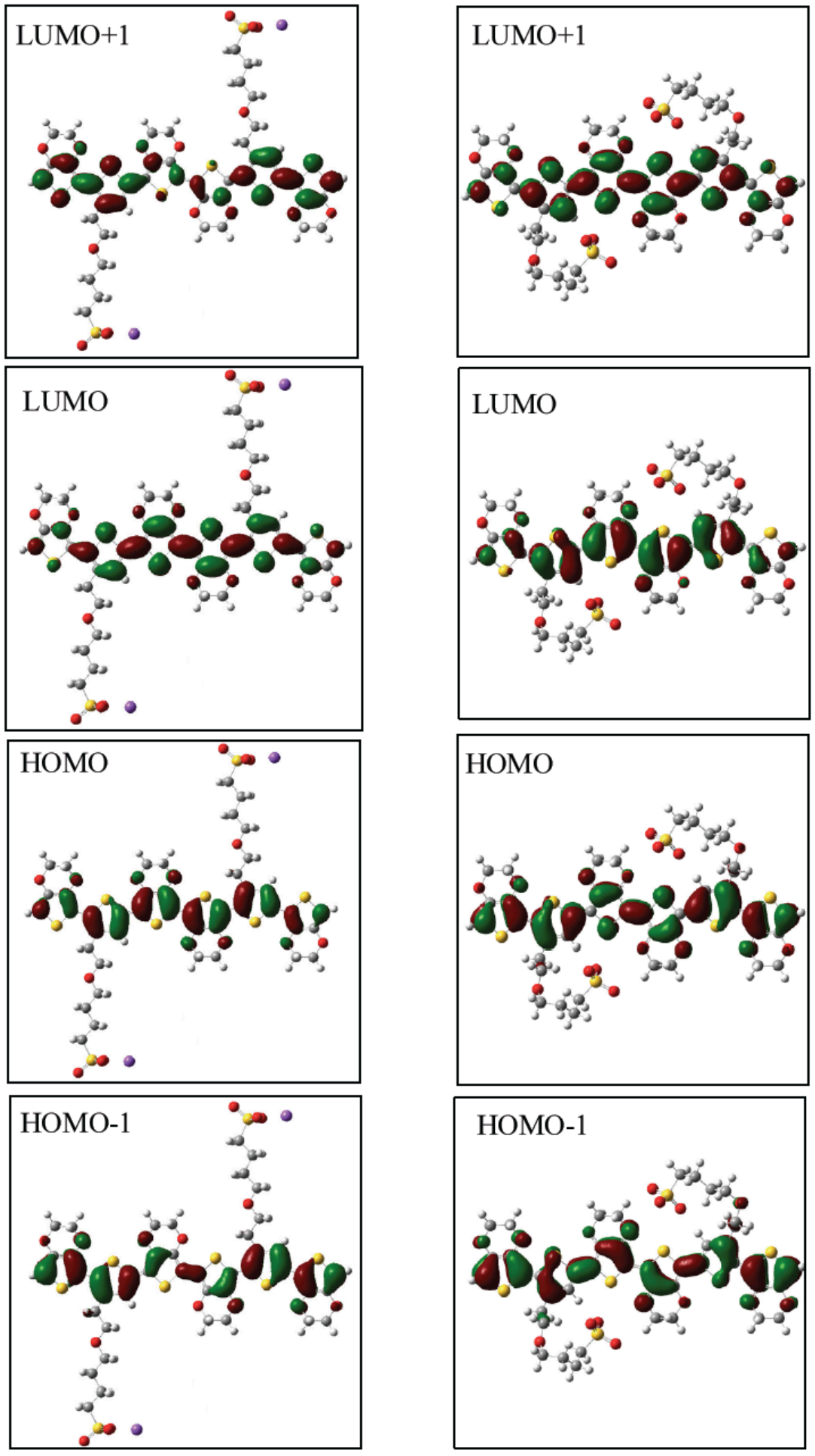

Figure S7. Main molecular orbitals involved (see Tables S1) in the ground state to the excited states transitions for oligomer in reduced state (left column) and bipolaron state (right column) with $n=2$ ETE-S units. 


\section{S1. Cyclic voltammetry}

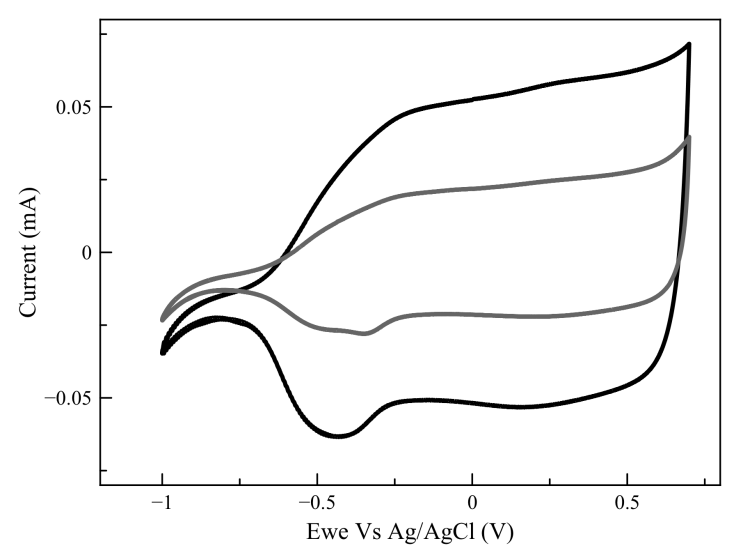

Figure S8. Cyclic voltammetry of ETE-S electropolymerized film with scanning rates of $20 \mathrm{mV} / \mathrm{s}$ (grey) and $50 \mathrm{mV} / \mathrm{s}$ (black).

The redox switching characteristics of conducting polymers (i.e transition from reduced to electrically conductive oxidized states) is commonly studied by cyclic voltammetry[1-2]. Cyclic voltammograms (CV) of ETE-S electropolymerized film recorded in potential range $1.0 \mathrm{~V}$ to $+0.7 \mathrm{~V}$ at different scan rates $(20,50, \mathrm{mV} / \mathrm{s})$ in $0.01 \mathrm{M} \mathrm{NaCl}$ are shown in figure Figure S8. The CV of ETE-S film exhibits the characteristic rectangular shape in the range from $-0.5 \mathrm{~V}$ to $0.7 \mathrm{~V}$, indicating the presence of polaronic and bipolaronic charge carriers in polymer film. On forward potential scan the increasing of current level in potential range from $V \approx-0.7 \mathrm{~V}$ to $V \approx-0.25$ attributed to the injection of charge carriers into reduced polymer film and expulsion of cations film to the electrolyte. Accordingly, on the reverse scan the voltammogram shows one cathodic peak in the same potential range corresponding to cation incorporation and expulsion of charge carriers sequentially. Finally, the low negative current contribution in potential range from $V \approx-1 \mathrm{~V}$ to $V \approx-0.7$ indicate presence of reduced states and the reduction of residual dioxygen.

[1] G. A. Snook, P. Kao, A. S. Best, J. Power Sources, 2011, 196, 1-12.

[2] J. C. Carlberg, O. Inganäs, JACS 1997, 144, L61-L64. 